\title{
Clustering of fMRI data: the elusive optimal number of clusters
}

\author{
Mohamed L Seghier ${ }^{\text {Corresp. } 1}$ \\ ${ }^{1}$ Cognitive Neuroimaging Unit, Emirates College for Advanced Education, Abu Dhabi, United Arab Emirates \\ Corresponding Author: Mohamed L Seghier \\ Email address: mseghier@gmail.com
}

Model-free methods are widely used for the processing of brain fMRI data collected under natural stimulations, sleep, or rest. Among them is the popular fuzzy c-mean algorithm, commonly combined with cluster validity (CV) indices to identify the 'true' number of clusters (components), in an unsupervised way. CV indices may however reveal different optimal c-partitions for the same fMRI data, and their effectiveness can be hindered by the high data dimensionality, the limited signal-to-noise ratio, the small proportion of relevant voxels, and the presence of artefacts or outliers. Here, the author investigated the behaviour of seven robust CV indices. A new CV index that incorporates both compactness and separation measures is also introduced. Using both artificial and real fMRI data, the findings highlight the importance of looking at different compactness and separation measures, defined here as building blocks of CV indices, to depict a full description of the data structure, in particular when no agreement is found between CV indices. Overall, for $\mathrm{fMRI}$, it makes sense to relax the assumption that only one unique c-partition exists, and appreciate that different c-partitions (with different optimal numbers of clusters) can be useful explanations of the data, given the hierarchical organization of many brain networks. 
1

2 Clustering of fMRI data: the elusive optimal number of clusters

4

Mohamed L Seghier

6

Cognitive Neuroimaging Unit, ECAE, Abu Dhabi, United Arab Emirates.

$\underline{\text { Running title: }}$ Unsupervised clustering of fMRI data.

13

*Corresponding author: Dr. Mohamed L Seghier, Cognitive Neuroimaging Unit, Emirates 18 College for Advanced Education, PO Box: 126662, Abu Dhabi, United Arab Emirates. Email:

19 mseghier@gmail.com

20

21

22

23

24

25

26

27

Word count: Title: 62 characters; Abstract: 200 words; Main text (without references and 29 legends): 6650 words.

30 Illustrations: 1 Table and 8 Figures; 3 supplementary figures. 


\section{Abstract}

36 Model-free methods are widely used for the processing of brain fMRI data collected under

37 natural stimulations, sleep, or rest. Among them is the popular fuzzy c-mean algorithm, 38 commonly combined with cluster validity $(\mathrm{CV})$ indices to identify the 'true' number of clusters

39 (components), in an unsupervised way. CV indices may however reveal different optimal c40 partitions for the same fMRI data, and their effectiveness can be hindered by the high data

41 dimensionality, the limited signal-to-noise ratio, the small proportion of relevant voxels, and the 42 presence of artefacts or outliers. Here, the author investigated the behaviour of seven robust CV 43 indices. A new CV index that incorporates both compactness and separation measures is also 44 introduced. Using both artificial and real fMRI data, the findings highlight the importance of 45 looking at different compactness and separation measures, defined here as building blocks of CV

46 indices, to depict a full description of the data structure, in particular when no agreement is found 47 between $\mathrm{CV}$ indices. Overall, for fMRI, it makes sense to relax the assumption that only one 48 unique c-partition exists, and appreciate that different c-partitions (with different optimal

49 numbers of clusters) can be useful explanations of the data, given the hierarchical organization of 50 many brain networks. 
55 Introduction:

56

57 58

There are many contexts where model-based methods are inadequate to map brain function, including for instance tasks that cannot be fully controlled (e.g. sleep, learning, natural stimulation, continuous rest) (e.g. (Bartels \& Zeki 2004; Bartels \& Zeki 2005; Hasson et al. 2004; Lee et al. 2012; Malinen et al. 2007; Zacks et al. 2001)) or when the hemodynamic correlates of neural activity are altered in unknown ways (e.g. patients with impaired vasculature). In such cases, approaches without a priori knowledge, known also as model-free or data-driven methods, are of great help.

Several data-driven methods have previously been used in fMRI (DonGiovanni \& Vaina 2016; Thirion et al. 2014), including fuzzy clustering (Baumgartner et al. 1998; Fadili et al. 2000; Golay et al. 1998; Jahanian et al. 2004) and independent component analysis (McKeown et al. 1998). These methods have been used in many scenarios and showed to be useful in extracting meaningful information from $\mathrm{fMRI}$ data in the absence of any prior knowledge (Aljobouri et al. 2018; Baumgartner et al. 2000; Lange et al. 2004; Ma et al. 2011; Smolders et al. 2007; Tang et al. 2015; Wismuller et al. 2004). One popular data-driven clustering method is based on the classic fuzzy c-mean (FCM) algorithm (Bezdek 1981). Although FCM allows high computational flexibility, its robustness may depend on several methodological issues. Specifically, these include the initialisation problem, the choice of similarity or distance metric, and the usually unknown optimal number of classes or prototypes (e.g. (Alexiuk \& Pizzi 2004; Esposito et al. 2002; Fatemizadeh et al. 2009; Jahanian et al. 2005; Lange et al. 2004; Moller et al. 2002; Quiqley et al. 2002; Soltanian-Zadeh et al. 2004; Windischberger et al. 2003)). This study focuses on the issue of the optimal number of clusters that can be extracted from fMRI data.

It is critical for any reliable clustering method to be able to determine whether (i) the data contains any structure and (ii) the segregated clusters are 'true' representations of the data (Dubes 1987; Windham 1981). This issue is generally expressed in terms of the ability of the algorithm, here FCM, to cluster the data into an optimal number of clusters $\left(\mathrm{c}_{\mathrm{opt}}\right)$. To do that, previous studies have introduced many measures, called cluster validity $(\mathrm{CV})$ indices, to estimate $\mathrm{c}_{\mathrm{opt}}$ in an unsupervised manner (for a review see (Bezdek \& Pal 1998; Hammah \& Curran 2000;

Kim \& Ramakrishna 2005; Maulik \& Bandyopadhyay 2002; Wang \& Zhang 2007; Zhou et al. 2014a)). The rationale behind these CV indices is that a good and useful clustering should yield 
86 compact and well-separated clusters. Indeed, it is not surprising that many proposed CV indices

87 combine different measures of compactness (cohesiveness) and separation (isolation) among 88 clusters, and would reach their optimal values for the best c-partition (i.e. data clustered into $\mathrm{c}_{\mathrm{opt}}$ 89 clusters).

90 A few studies have previously investigated the effectiveness of CV indices in the context of 91 fMRI data clustering (e.g. (Alexiuk \& Pizzi 2004; Fadili et al. 2000; Fadili et al. 2001; Goutte et 92 al. 1999; Moller et al. 2002; Seghier \& Price 2009)). Some known features of fMRI data may 93 make the clustering particularly challenging (Thirion et al. 2014), including for instance the huge 94 number of points (i.e. voxels) in a typical fMRI dataset, the poor signal-to-noise ratio in fMRI 95 (noisy data), the small proportion of voxels of interest that might be considered as relevant (i.e. 96 an ill-balanced problem), and the presence of artefacts or outliers (i.e. caused by head motion or 97 signal loss). Given this complexity, it might the case that reliance on a single CV index might not 98 be enough, in particular when the data are noisy and the expected number of clusters is relatively 99 high. Here, the author compared the identified optimal c-partition when applying different CV 100 indices to the same datasets. In particular, the author investigated the behaviour of different 101 measures of compactness and separation when using previously published CV indices. The 102 current study also aims to introduce a new CV index that specifically incorporates suitable 103 compactness and separation measures that are useful for data with larger optimal number of 104 clusters. 
108 Methods:

109

\section{Fuzzy clustering}

111 Our clustering method was based on the popular fuzzy c-mean (FCM) algorithm (Bezdek 1981;

112 Bezdek et al. 1997). In the context of fMRI, the FCM algorithm can segregate or cluster $n$ brain

113 voxels (feature vectors) into $c$ expected clusters $(\mathrm{c} \geq 2)$. Each voxel $i$ is a vector $X_{i}$ of $p$ properties

114 (e.g. number of collected volumes or scans). Each cluster $j$ is characterised by a centroid $V_{j}$, that

115 represents its characteristic timecourse (prototype). The resemblance between each voxel $i$ and

116 each centroid $V_{j}$ is assessed by the distance $D_{i j}$ between $X_{i}$ and $V_{j}$. The degree of membership $U_{i j}$

117 is calculated for each voxel $i$ by comparing $D_{i j}$ for each cluster $j$ to all other clusters.

118 In brief, the standard FCM algorithm iteratively minimises the following objective function $J_{\mathrm{m}}$ :

$119 J_{m}=\sum_{i=1}^{n} \sum_{j=1}^{c} U_{i j}^{m} \cdot D_{i j}^{2}$

120 where " $m$ " is the degree of fuzziness.

121 Degrees of membership $U$ and centroids $V$ are updated as following:

122

$U_{i j}=\frac{1}{\sum_{k=1}^{c}\left(\frac{D_{i j}}{D_{i k}}\right)^{2 / m-1}}$

$V_{j}=\frac{\sum_{i=1}^{n} U_{i j}^{m} \cdot X_{i}}{\sum_{i=1}^{n} U_{i j}^{m}}$

124 Optimal clustering depends on the choice of the similarity $D$, the degree of fuzziness $m$ and the 125 optimal number of clusters $c_{\text {opt }}$, as detailed below.

126

\section{Similarity measure D}

128 Here I used a modified version of the hyperbolic correlation distance proposed previously by

129 Golay and colleagues (Golay et al. 1998). In their work, $D$ was defined as (Golay et al. 1998):

$130 \quad D_{i j}=\frac{1-C C_{i j}}{1+C C_{i j}}$

131 Where $C C_{i j}$ is the Pearson correlation coefficient between $X_{i}$ and $V_{j}$. 
132 Here, a modified version of $D$ was used:

$133 D_{i j}=\frac{\sqrt{\left|C C_{i j}\right|}-C C_{i j}}{\sqrt{\left|C C_{i j}\right|}+C C_{i j}}$

134 This new formula uses the square root function, a monotonically increasing function over $\mathrm{x}>0$

135 that satisfies the following inequality: $\sqrt{x} \geq x$, for $\mathrm{x} \in[0,1]$. The rationale here was to increase the

136 difference (i.e. discrimination power) between relatively close correlation values in particular

137 between mid and high correlations (cf. Supplementary Figure 1).

138

139 Optimal number of clusters

140 A good and robust clustering should yield compact and well-separated clusters. This is assumed

141 to be the case when the number of clusters reaches an optimal value $c_{\text {opt }}$. The exact $c_{\text {opt }}$ value is

142 however unknown in fMRI data. Previous reports have suggested that $\mathrm{c}_{\mathrm{opt}}$ can be found within

143 the interval $\left[2, \sqrt{n}\right.$ ] (Zahid et al. 1999b); however the exact $\mathrm{c}_{\mathrm{opt}}$ can only be estimated

144 empirically. Typically, FCM is repeated several times with different $c$ values (i.e. equivalent to

145 an unsupervised fuzzy clustering analysis (Fadili et al. 2001)) and the $c$ value that optimises a

146 given criterion, here a given $\mathrm{CV}$ index, is considered as the optimal $\mathrm{c}_{\mathrm{opt}}$, and that criterion is

147 typically defined as a trade-off between compactness and separation.

148 Before introducing the different $\mathrm{CV}$ indices used here, it might be helpful to define the core

149 measures of compactness and separation using unified mathematical notations. These measures

150 can be seen as building blocks that can be combined into different CV indices. Ultimately, the

151 definition of those measures would help appreciate the inherent links (or similarity) between

152 previously suggested $\mathrm{CV}$ indices, before introducing the rationale of the new $\mathrm{CV}$ index.

153

\section{Compactness and separation measures}

155 Two core quantities, noted $n_{m, j}$ and $\sigma_{m, j}$, were defined as following:

$156 \quad n_{m, j}=\sum_{i=1}^{n} U_{i j}^{m}$

$157 \quad \sigma_{m, j}=\sum_{i=1}^{n} U_{i j}^{m} \cdot D_{i j}^{2}$ 
158 The measures $n_{1, j}$ and $n_{2, j}$ represent the fuzzy cardinality and the fuzzy partition of cluster $j$

159 respectively. The quantity $\sigma_{m, j}$ denotes the fuzzy variation of cluster $j$, though other studies have

160 instead used $\sigma_{1, j}$ as a measure of fuzzy variation (e.g. (Gath \& Geva 1989; Rezaee et al. 1998;

161 Sun et al. 2004)).

162

163 Those core quantities can then be combined into different forms to give away different measures

164 of fuzzy compactness (cohesiveness) for a given c-partition. Using similar notation as previous

165 studies, quantities called $\pi_{m, 1}$ (Bensaid et al. 1996; Zahid et al. 1999a), $\pi_{m, m}$ (Bouguessa et al.

166 2006), and FC (Fadili et al. 2001; Zahid et al. 1999a) were computed as following:

$167 \pi_{m, 1}=\sum_{j=1}^{c} \frac{\sigma_{m, j}}{n_{1, j}}$

$168 \pi_{m, m}=\sum_{j=1}^{c} \frac{\sigma_{m, j}}{n_{m, j}}$

$169 F C=\frac{\sum_{i=1}^{n}\left(\max _{j}\left(U_{i j}\right)\right)^{2}}{\sum_{i=1}^{n} \max _{j}\left(U_{i j}\right)}$

170

171 Likewise, the fuzzy separation (isolation) between clusters was previously estimated with several

172 fuzzy separations quantities called $K_{m}$ (Fukuyama \& Sugeno 1989), FS (Fadili et al. 2001; Zahid

173 et al. 1999a), $S$ (Zahid et al. 1999a) and $S S$ (Rezaee et al. 1998):

174

$K_{m}=\sum_{j=1}^{c} n_{m, j} \cdot\left\|V_{j}-\bar{X}\right\|^{2}$

$175 \quad F S=\sum_{j=1}^{c-1} \sum_{k=1}^{c-j} \frac{\sum_{i=1}^{n}\left(\min \left(U_{i j}, U_{i, k+j}\right)\right)^{2}}{\sum_{i=1}^{n} \min \left(U_{i j}, U_{i, k+j}\right)}$

$176 \quad S=\frac{1}{c} \sum_{j=1}^{c}\left\|V_{j}-\bar{X}\right\|^{2}$ 
$177 \quad S S=\sum_{j=1}^{c} \frac{1}{\sum_{k=1}^{c}\left\|V_{j}-V_{k}\right\|}$

178 where $\bar{X}$ stands for the global mean of the whole data.

179 Interestingly, the ratio $F S / F C$ (i.e. separation divided by compactness) is known as the fuzzy

180 overlap (FO) coefficient (see (Fadili et al. 2000) for more details).

181

182 Furthermore, different measures of between-centroid distance have been proposed, including the

183 minimum distance $V_{d \text { min }}$ (e.g. (Schwämmle \& Jensen 2010; Xie \& Beni 1991)), the maximum

184 distance $V_{d \max }$ (e.g. (Rezaee et al. 1998)), and the minimum distance $V_{d \min , j}$ between a cluster $j$

185 and the remaining clusters (Wu \& Yang 2005):

$186 \quad V_{d \min }=\min _{j, k}\left(\left\|V_{j}-V_{k}\right\|\right)$

$187 \quad V_{d \max }=\max _{j, k}\left(\left\|V_{j}-V_{k}\right\|\right)$

$188 V_{d \min , j}=\min _{k \neq j}\left(\left\|V_{j}-V_{k}\right\|\right)$

189 These measures, based on the distance between estimated centroids, can be seen as alternative

190 separation measures. They can be handy when the clustering is showing redundant clusters.

191

192 This section introduces two new measures of separation and discrimination between voxels by

193 combining different measures of fuzzy cardinality and variation (cf. Equations 6-7): a fuzzy

194 intra-cluster $\left(I D_{\text {int } r a}\right)$ dissimilarity coefficient and an inter-cluster $\left(I D_{\text {inter }}\right)$ dissimilarity

195 coefficient:

$196 I D_{\text {int } r a}=\max _{j}\left(\frac{n-n_{1, j}}{n_{1, j}} \cdot \frac{\sigma_{1, j}}{\sum_{k=1, k \neq j}^{c} \sigma_{1, k}}\right)$

$197 I D_{\text {inter }}=\min _{j}\left(\frac{\min _{k, k \neq j}\left(\sigma_{1, k}\right)}{\sigma_{1, j}}\right)$

198 Small $I D_{\text {intra }}$ values would indicate that, across all clusters, voxels that are close to a given

199 cluster are well-isolated from voxels that are far from that cluster, whereas high $I D_{\text {inter }}$ values 
200 indicate well-discriminated voxels (i.e. small fuzzy overlap between clusters). Our initial tests

201 with noisy simulated data showed the need to define new separation measures that are robust to 202 noise and can handle c-partitions with higher number of clusters, hence the new definitions in 203 Equations (18) and (19).

204

205

\section{Cluster validity measures}

206 There are many $\mathrm{CV}$ indices in the literature (probably more than 50 indices), hence it is beyond

207 the scope of this study to test all of them. In a preliminary analysis (results not shown here), 208 about 20 selected $\mathrm{CV}$ indices were first tested on several simulated datasets (as defined in 209 (Bezdek \& Pal 1998; Bouguessa et al. 2006; Dave 1996; Fukuyama \& Sugeno 1989; Geva et al. 210 2000; Kim et al. 2001; Kim et al. 2003; Kim \& Ramakrishna 2005; Kwon 1998; Pakhira et al. 211 2004; Pakhira et al. 2005; Pal \& Bezdek 1995; Rezaee et al. 1998; Rhee \& Oh 1996; Sun et al. 212 2004; Tsekouras \& Sarimveis 2004; Wu \& Yang 2005; Xie \& Beni 1991; Yu \& Li 2006; Zahid 213 et al. 1999a; Zahid et al. 1999b)). These CV indices were selected from earlier studies (for a 214 similar rationale, see recent comparison study (Zhou et al. 2014a)), and many of them are well215 established indices. Some of these CV indices have been used in previous fMRI studies. More 216 recent CV indices (e.g. see (He et al. 2016; Hu et al. 2011; Lin et al. 2016; Ren et al. 2016;

217 Rezaee 2010; Yang et al. 2018; Zhang et al. 2014)) were not explicitly tested here.

218 From this preliminary analysis, seven CV indices (out of twenty) were selected according to the 219 following four criteria: CV indices should (i) combine both measures of separation and 220 compactness; (ii) not suffer from monotonic dependency with the number of expected clusters;

221 (iii) not necessitate the categorisation or the binarisation of $U$ (i.e. crisp degrees of membership)

222 during $\mathrm{CV}$ computation; (iv) be fast to compute when $n$ is expected to be very high (e.g.

223 hundreds of thousands of voxels in the context of fMRI data). The seven selected CV indices that 224 satisfied the different criteria are described below and listed in Table 1.

1)- The Rezaee-Lelieveldt-Reider index $C V_{R L R}$ (Rezaee et al. 1998): 
$229 \quad C V_{R L R}=\frac{\sum_{j=1}^{c} \sigma_{1, j}}{c \cdot\left\|\sigma_{X}\right\|}+\frac{1}{\alpha} \cdot\left(\frac{V_{d \max } \cdot S S}{V_{d \min }}\right)$

230 The constant $\alpha$ is a weighting constant and $\sigma_{X}$ is the variance of the whole data set. The best c-

231 partition is obtained by minimising $C V_{R L R}$ with respect to the number of clusters $c$. In the

232 original definition of $C V_{R L R}$, the constant $\alpha$ was set to 1; however, here $\alpha$ was set to the value of

$233 \frac{V_{d \max }}{V_{d \min }} \cdot S S$ at the maximum number of clusters $\left(\mathrm{c}_{\max }\right)$ as suggested previously (Sun et al. 2004).

234

235 2)- The Zahid-Limouri-Essaid index $C V_{Z L E}$ (Zahid et al. 1999a; Zahid et al. 1999b):

$236 C V_{Z L E}=\alpha \cdot\left(\frac{S}{\pi_{m, 1}}\right)-\frac{F S}{F C}$

237 The constant $\alpha$ is independent from $\mathrm{c}$ and was introduced here as a scaling factor to take into

238 account the difference in values between the two subtracted quantities. The constant $\alpha$ was set

239 here to the value of the fuzzy overlap (FS/FC) at $\mathrm{c}=\mathrm{c}_{\max }$ (note that in the original paper of Zahid

240 et al., $\alpha$ was set equal to 1). The best c-partition is obtained by maximising $C V_{Z L E}$ with respect to

241 c. This $C V_{Z L E}$ index has previously been used for fMRI analysis (Fadili et al. 2001).

242 Note that the ratio $\left(\frac{S}{\pi_{m, 1}}\right)$ in Equation (21) is also known as the Pal-Bezdek cluster validity

243 index (Pal \& Bezdek 1995).

244

245 3)- Among several CV indices suggested by Geva and colleagues (Geva et al. 2000), the

invariant index $C V_{G V}$ was selected here to measure the ratio of the between-cluster scatter matrix

to the within-cluster scatter matrix (Geva et al. 2000):

$248 \quad C V_{G V}=\frac{K_{1}}{c^{2} \cdot J_{1}}$

249 The normalisation with the number of clusters $c$ minimise the monotonically increase of $C V_{G V}$

250 when $c$ increased. This index should be maximal at the optimal c-partition. 
252 4)- The Kim-Park index, noted $C V_{K P}$ (Kim et al. 2001):

$253 \quad C V_{K P}=\frac{\pi_{1,1}}{c}+\frac{1}{\alpha} \cdot\left(\frac{c}{V_{d \text { min }}}\right)$

254 The best c-partition is obtained by minimising the index $C V_{K P}$ with respect to the number of

255 clusters $c$. This index has previously been used for fMRI analysis (Moller et al. 2002).

256

257 5)- The Pakhira-Bandyopadhyay-Maulik index $C V_{P B M}$ (Pakhira et al. 2004; Pakhira et al. 2005):

$258 C V_{P B M}=\frac{\alpha}{c} \cdot \frac{V_{d \max }}{J_{m}}$

259 With $\alpha$ as a constant term (e.g. $\alpha$ was set here to $n$ ). The best c-partition is obtained by

260 maximising $C V_{P B M}$ with respect to the number of clusters $c$.

261

262 6)- The Wu-Yang index $C V_{W Y}$ (Wu \& Yang 2005):

$263 C V_{W Y}=\sum_{j=1}^{c}\left(\frac{n_{1, j}}{\max _{j}\left(n_{1, j}\right)}-\exp \left(-\frac{V_{d \min , j}^{2}}{S}\right)\right)$

264 This index compared the fuzzy partition of each cluster to its exponential separation, with $265-c<C V_{W Y}<c$, and $C V_{W Y}$ is maximal at $\mathrm{c}_{\mathrm{opt}}$.

267 7)- The Bouguessa-Wang-Sun index $C V_{B W S}$ index (Bouguessa et al. 2006):

$268 C V_{B W S}=\frac{K_{m}}{\pi_{m, m}}$

269 This index $C V_{B W S}$ should be maximised with respect to c.

270

271 8)- Our new CV index, noted $C V_{\text {new }}$, combined different measures of compactness and 272 separation as following:

273

$274 C V_{\text {new }}=K_{m} \cdot\left(\frac{I D_{\text {inter }}}{I D_{\text {int } r a}}\right) \cdot\left(\frac{F C}{J_{1}}\right)$ 
276 The best c-partition should maximise $C V_{\text {new }}$. The rationale behind incorporating those specific

277 compactness and separation measures ( $\left.I D_{\text {inter }}, K_{m}, F C, I D_{\text {intra }}, J_{1}\right)$ in the definition of $C V_{\text {new }}$ is

278 illustrated below with simulated (noisy) datasets.

279

280 Simulated data

281 Twenty-two simulated datasets were generated as following. First, a fixed number $c$ of time282 courses with $p$ datapoints $(p=100)$ were generated from a unit normal distribution (mean $=0, \sigma=$

283 1). The Pearson correlation between these $c$ time-courses was less than 0.1 for all simulated

284 datasets. Second, each time-course was replicated $\mathrm{r}_{\mathrm{j}}$ times, with $\mathrm{j}=1 \ldots \mathrm{c}_{\mathrm{opt}}$, and $\sum_{j=1}^{c} r_{j}=n$ (where $n$

285 is the total number of voxels, set here to 1000). Third, $n$ random timecourses with $p$ datapoints,

286 generated from a normal distribution $($ mean $=0)$ but with variable noise levels $(\sigma=1$ or 4$)$ were

287 added to the replicated time-courses. This would help to test the robustness of FCM at different

288 noise levels (for a similar rational see (Kim et al. 2001; Wang \& Zhang 2007)) and to monitor

289 the behaviour of the different $\mathrm{CV}$ indices when the fuzzy compactness of clusters became very

290 low (i.e. high intra-class dissimilarity in noisy data). This procedure generated a dataset $X$ of $n$

291 voxels, each with $p$ datapoints, with known and fixed numbers of classes. Multidimensional

292 scaling (MDS) tools were used to visualise the simulated $c$ clusters.

293 Specifically, the following 22 datasets were generated: (i) a single-cluster dataset (noted 1-

294 cluster; e.g. the 'null' case, see (Tibshirani et al. 2001)) with highly similar voxels $\left(\mathrm{c}_{\mathrm{opt}}=1\right.$;

295 Supplementary Figure 2a); (ii) a dataset without any obvious structure (noted $n$-cluster data; $\mathrm{c}_{\text {opt }}$ 296 near to $n$; Supplementary Figure 2b), see (Suleman 2017); (iii) ten datasets with known number

297 of clusters $\mathrm{c}_{\mathrm{opt}}$ varying from 2 to 11 and low noise level ( $\sigma=1$, see illustration in Supplementary

298 Figure $2 \mathrm{c}$ with $\mathrm{c}_{\mathrm{opt}}=3$ ); (iv) ten datasets with a known number of clusters $\mathrm{c}_{\mathrm{opt}}$ varying from 2 to

29911 and high noise level $\left(\sigma=4\right.$, see illustration in Supplementary Figure $2 d$ with $\left.\mathrm{c}_{\mathrm{opt}}=3\right)$.

300 All simulated datasets were clustered by FCM with $c$ (i.e. number of expected clusters) varying

301 between $c_{\min }=2$ and $c_{\max }=19$. All analyses were carried out with homemade Matlab-based scripts

302 (MathWorks, Natick, Massachusetts USA).

303

Real fMRI data 
305 Real data consisted of single subject fMRI data with a block paradigm design (freely available

306

307

308

309

310

311

312

313

314

315

316

317

318

319

320

321

322

323

324

325

326

327

\section{Degree of fuzziness $\mathbf{m}$}

329

330

331

332

333

334

335 regions) were calculated.

at: http://www.fil.ion.ucl.ac.uk/spm/data/auditory.html). The block paradigm consisted of alternated epochs between rest and auditory stimulation. 96 volumes were acquired on a modified 2T Siemens MAGNETOM Vision system (TR=7s, 64 contiguous slices). To avoid $\mathrm{T} 1$ effects in the initial scans, the first 12 scans were discarded, leaving 84 scans for further analysis $(p=84)$. The data were realigned, normalised (voxel size $2 \times 2 \times 2)$ and smoothed (FWHM $=6 \times 6 \times 6$ $\mathrm{mm})$. This dataset was selected because it has been used in many previous studies with clustering techniques including FCM (e.g. (Gu et al. 2005; Lu et al. 2004)). FCM was applied on this real fMRI dataset with $c$ varying between $\mathrm{c}_{\min }=2$ and $\mathrm{c}_{\max }=39$. To identify relevant FCM cluster(s) with activated auditory regions, the centroids (prototype) $V_{j}(\mathrm{j}=1 \ldots \mathrm{c})$ were correlated with the experimental block design (Bandettini et al. 1993).

To appreciate the distribution of brain regions' sizes in each c-partition, a morphological granulometry was applied to all identified clusters after binarization (Soille 2003). This analysis estimated the size of each spatially distinct region or blob (26-connected neighbourhood) for a given crisp FCM partition. Given that each voxel belongs to all clusters at different degrees of membership (cf. $U_{i j}$ in Equation (2)), the threshold was set to 0.5 so that each voxel belongs maximally to one cluster. Practically, for a given c-partition and for each binary cluster $j$ $(j=1 \ldots c)$, the size of each region as well as the number of isolated voxels (i.e. single-voxel

This dataset was also analysed with SPM12 software package (Wellcome Trust Centre for Neuroimaging, London UK, http://www.fil.ion.ucl.ac.uk/spm/) using standard procedures. This allowed auditory activations to be identified using model-based methods.

The degree of fuzziness $m$ might influence the output of clustering (e.g. (Bezdek 1981; Fadili et al. 2000; Fadili et al. 2001; Krishnapuram \& Keller 1993; Selim \& Ismail 1986; Yu et al. 2004)): when $\mathrm{m}$ tends to 1 the classification becomes crisp and $U_{i j}$ takes the value 0 (voxel $i$ is not a member of cluster $j$ ) or 1 (voxel $i$ belongs to cluster $j$ ) but when $\mathrm{m}$ tends to $+\infty$ the classification is purely fuzzy $\left(U_{i j}\right.$ is near to $\left.1 / c\right)$. The optimal value of $m$ may depend on the characteristics of the data. Previous empirical work approximated $m$ by a nonlinear function of the dimensions of the data ( $n$ and $p$ ); for example, Equation (5) of Schwammle and Jensen (2010, Page 2845) yields 
$336 \mathrm{~m}$ values of 1.044 and 1.019 for our artificial and real datasets respectively. However, these

337 estimated values are too low compared to typical $m$ values encountered in neuroimaging studies.

338 Previous studies have explored the influence of $m$ on the computation of CV indices (e.g. (Zhou

339 et al. 2014b)), and they found better clustering results with $m$ between 1.2 and 2.5 for fMRI data

340 (Fadili et al. 2000; Fadili et al. 2001; Moller et al. 2002; Smolders et al. 2007).

341 More specifically, there are two issues to be considered when selecting $m$ during the computation

342 of $\mathrm{CV}$ indices. First, several $\mathrm{CV}$ indices became inadequate with hard c-partitions (i.e. $m$ tends to

343 1). Specifically, any measures that are based exclusively on the distribution of $U$ values (e.g. $F C$,

$344 F S$ ) would artificially reach their optimal values independently from the number of clusters $c$.

345 Second, according to Equation (3), centroids become close to the mean of the whole data set $\bar{X}$

346 when $m$ tends towards $+\infty$. In other words, the $c$ clusters would have comparable fuzzy

347 cardinality values (i.e. Equation (6)) for larger $m$ values, which may be problematic when some

348 clusters are expected to contain a small number of voxels (see illustration in Supplementary

349 Figure 3); for more details see (Selim \& Ismail 1986; Tsekouras \& Sarimveis 2004; Yu et al.

350 2004). This issue is particularly critical when analysing task-related fMRI data because activated

351 voxels are expected to represent a small fraction of the whole brain.

352 Here, $m$ was held to 1.5 throughout this study.

353

354 Voxel selection and the ill-balanced dataset problem in fMRI

355 One important issue during the clustering of fMRI datasets is the selection of the relevant $n$

356 voxels. Because the number of activated voxels is small (i.e. a few percent) compared to the total

357 number of voxels in a typical whole-brain fMRI dataset, previous studies have suggested

358 different approaches to overcome this 'ill-balanced' data problem. To do that, previous studies

359 limited FCM to relevant voxels within the gray matter, in specific anatomical brain regions, or to

360 voxels with some kind of task-related effects (e.g. see ((Fadili et al. 2000; Goutte et al. 1999; Gu

361 et al. 2005; Lee et al. 2012; Moller et al. 2002; Seghier et al. 2007)). Voxel selection might be

362 useful for (i) reducing the high dimensionality of the problem and improving both computational

363 robustness and speed; (ii) minimising the influence of redundant voxels; and (iii) increasing the

364 accuracy of the clustering by focusing mainly on meaningful voxels. However, the author

365 preferred here to include all brain voxels so that the robustness of the different CV indices can be

366 appreciated when noisy voxels (voxels with no effect of interest) and artefacts are present. FCM 
367 was thus applied to all voxels of the real fMRI dataset, yielding a total number of voxels $n=$ $368227,716$.

369

370 FCM convergence and the initialisation problem

371 Depending on the initialisation of the degrees of membership $U$ (Bezdek 1981), the FCM

372 algorithm may converge to different c-partitions (e.g. local minima). This problem of

373 initialisation may lead to spurious c-partitions (Moller et al. 2002) when using CV indices. One

374 possible solution to that problem is to repeat the FCM algorithm on the same dataset with several

375 different random initialisations (e.g. (Moller et al. 2002; Pena et al. 1999)), with the expectation

376 that it is unlikely that different starting conditions will lead to the same local minima.

377 Accordingly, the FCM algorithm was re-run on the real fMRI dataset ten times with random

378 initialisations (for a similar procedure see (Chuang et al. 1999)).

379 
380 Results:

381

382

\section{FCM on simulated data}

383

384

\section{The 1-cluster dataset}

385 Clustering the 1-cluster dataset $\left(\mathrm{c}_{\mathrm{opt}}=1\right)$ showed how compactness and separation measures behave when data cannot be clustered any further. In this context of high redundancy, it is expected to observe: (i) high similar or identical centroids $V$, (ii) degrees of membership $\mathrm{U}$ near to the fuzziest value $1 / \mathrm{c}$, and (iii) comparable fuzzy cardinality across clusters. As illustrated in Figure 1A, the fuzzy compactness $F C$ decreased monotonically with $c$ (i.e. $F C=1 / \mathrm{c}$ ) whereas fuzzy separation $F S$ increased linearly with $c$-1 (i.e. $F S=(\mathrm{c}-1) / 2$ ), suggesting a high fuzzy overlap $F O$ between clusters. Likewise, as expected, the fuzzy compactness $\pi_{m, 1}$ and separation $K_{m}$ showed monotonic dependency with $c^{m-1}$ and $c^{1-m}$ respectively, suggesting that the product $\pi_{m, 1} \cdot K_{m}$ remained constant (independent from $c$ ) when data were classified into pure fuzzy

394 clusters. Interestingly, measures of separation based on centroids $V\left(\right.$ e.g. $\left.\mathrm{V}_{\mathrm{dmin}}, \mathrm{V}_{\mathrm{dmax}}, \mathrm{S}\right)$ and distances $D$ (e.g. $I D_{\text {intra }}$ and $I D_{\text {inter }}$ ) were independent from $c$, suggesting highly similar (i.e. identical) centroids $V$.

The n-cluster dataset

401 Clustering the $n$-cluster dataset (i.e. $\mathrm{c}_{\text {opt }}$ towards $n$ ) tested the robustness of the different

402 measures of compactness and separation when data is patternless with high dispersion.

403 Compactness coefficients showed similar behaviour as above when clustering the 1-cluster 404 dataset, except for $J_{1}$ and $S S$ measures. Interestingly, separation measures based on centroids $V$ 405 and distances $D$ showed more complex dependencies with $c$ (Figure 1B) as compared to the 1406 cluster case (Figure 1A), in particular when using the two new coefficients $I D_{\text {intra }}$ and $I D_{\text {inter }}$. 407

408 What emerged from above is that $K_{m}, I D_{\text {intra }}, I D_{\text {inter }}$, and $J_{1}$ behaved differently on 1-cluster and $409 n$-clusters datasets, which is highly desirable when clustering fMRI data that have complex 
410 structure. These results motivated the rationale of including them in the computation of the new $411 C V_{\text {new }}$ index (as defined in Equation (27)).

412

\section{CV indices on data with known numbers of clusters}

414 The different measures of compactness and separation are shown in Figure 2A for the 7-clusters 415 data set. Several measures showed different values over the number of clusters as compared to 416 the clustering of the 1 -cluster and $n$-cluster datasets. For instance, the coefficient $J_{1}$ decreased in 417 the interval $c=2$ to $c=7$, consistent with the fact that data can be clustered further (as seen for the $418 n$-cluster data); then it reached a plateau for higher number of classes, consistent with the fact 419 that the data cannot be segregated any further (as the case of the 1-cluster data). The limit 420 between the two behaviours was indeed at the true number of clusters $(c=7)$. This observation is 421 valid for the other measures of compactness (e.g. $F C, \pi_{m, 1}, I D_{\text {int } r a}$ ) and separation (e.g. $F S$, $\left.422 I D_{\text {inter }}, \mathrm{S}, K_{m}\right)$. When the data became noisy, some measures were less sensitive to the structure 423 of the data (i.e. the presence of 7 clusters). As illustrated in Figure 2B, fuzzy separation $F S$ and 424 compactness $\pi_{m, 1}$ showed comparable behaviour as in the clustering of the $n$-cluster dataset, which reflects the influence of noisy distant points (low within-cluster compactness and between-cluster separation). Interestingly, in addition to $V_{d m i n}$, quantities $I D_{\text {int } r a}, K_{m}$ and $J_{1}$ were more robust to noise and showed high discriminability with an optimal value around the expected number of classes (Figure 2B). This observation further motivated their inclusion in the definition of the new CV index.

Figure 3 illustrates all CV indices for the 3-cluster, 7-cluster, and 11-cluster datasets with low noise level $(\sigma=1)$. All CV indices indicated the best c-partition for the expected number of clusters (maximum value for $C V_{Z L E}, C V_{G V}, C V_{P B M}, C V_{W Y}, C V_{B W S}, C V_{n e w}$; minimum value for $C V_{R L R}$ and $\left.C V_{K P}\right)$. Note that the new index $C V_{\text {new }}$ is highly discriminative in pointing to the 437 optimal c-partition. When the data became noisy $(\sigma=4)$, all $\mathrm{CV}$ indices, except $C V_{B W S}$ and $C V_{\text {new }}$ 438 , failed to indicate the optimal c-partition (Figure 4). However, for data with higher $\mathrm{c}_{\mathrm{opt}}$ (e.g. 
$439 \mathrm{c}_{\mathrm{opt}}>9$ ), only the new index $C V_{\text {new }}$ identified the true number of clusters, albeit with lower 440 discriminability (e.g. compare Figure 3B to Figure 4B).

441 An ad hoc analysis was conducted to monitor the behaviour of $C V_{\text {new }}$ over different degrees of

442 fuzziness $m$ ( $m$ varying between 1.2 and 2.5), for a similar rationale see (Schwämmle \& Jensen 443 2010). This analysis showed that $C V_{\text {new }}$ correctly identified the true number of clusters $\mathrm{c}_{\mathrm{opt}}$ in

444 almost all simulated datasets for $m \in[1.2,2.5]$, except for datasets with both high noise level $445(\sigma=4)$ and high number of true clusters $\left(\mathrm{c}_{\mathrm{opt}}>9\right)$ where $C V_{\text {new }}$ failed to identify $\mathrm{c}_{\mathrm{opt}}$ when $m \geq 2$ 446 (i.e. $C V_{n e w}$ underestimated $\mathrm{c}_{\mathrm{opt}}$ at higher $m$ values including the popular value of $m=2$ ). This ad 447 hoc analysis confirmed the initial choice of $m=1.5$.

\section{FCM real fMRI data}

As expected, the number of iterations for the convergence of the FCM algorithm varied across the 10 different initialisations. However, for a given $c$ value and across the ten runs, the obtained c-partitions were very similar and the function $J_{\mathrm{m}}$ (Equation (1)) reached the same minimum value (except for $c$ values between 12 and 15 where one initialisation reached a different minimal $J_{\mathrm{m}}$ value compared to the other nine initialisations).

\section{Identified clusters}

460 Figure 5 plots the different coefficients and $\mathrm{CV}$ indices against the number of expected clusters $c$ varying from 2 to 39. Measures such as $I D_{\text {inter }}$, and $V_{\text {dmin }}$ showed an interesting pattern when $c$ increased, with high and decreasing values for small number of clusters $(c<10)$ and low and fixed values when $c$ increased (a comparable behaviour was also seen for $F C$ ). This mirrored their behaviour during the clustering of the 1-cluster and n-cluster datasets. The change in the nature of the dependency occurred around $\mathrm{c}=13$, indicating the maximum $\mathrm{c}$ value that ensured different centroids V. For a number of expected clusters bigger than 13, the c-partition contained a few redundant classes (identical centroids $V$ ). However, for $\mathrm{c}<13$ clusters, although the 
469 optimal (see for instance $V_{\mathrm{dmax}}, S$, and $K_{m}$ ). More specifically, the fuzzy separation measures $S$

470 and $K_{m}$ showed optimal values for higher numbers of expected clusters at $c$ larger than 17

471 clusters. At this range, the c-partition contained at least three similar centroids.

472 Figure 5B illustrates the dependency of different CV indices with $c$. Some CV indices (e.g.

$473 C V_{R L R}$ and $C V_{K P}$ ) showed optimal values for low $c$ values (maximal fuzzy compactness),

474 whereas other $\mathrm{CV}$ indices (e.g. $C V_{Z L E}, C V_{B W S}, C V_{G V}$, and $C V_{P B M}$ ) showed optimal values at an

475 intermediate number of expected clusters (i.e. maximal fuzzy separation). Interestingly, the new 476 index $C V_{\text {new }}$ went through different phases (i.e. different plateaus), depending on the weight of

477 fuzzy separation and compactness (a change of behaviour visible at c=15). The new index $C V_{\text {new }}$

478 reached its maximum value at $c=24$ clusters, ensuring a good compromise between separation

479 and compactness of the c-partition of this real fMRI dataset.

480 The results of the morphological granulometry at $U>0.5$ are illustrated in Figure 6. As expected, 481 the size of very large regions tend to decrease with the number of expected clusters, as large

482 regions were subdivided further into smaller regions at higher c values. Interestingly, for each c483 partition, the total number of single-voxel regions over all clusters was less than $0.04 \%$ of $n$ 484 (Figure 6). Given the spatial smoothness of the fMRI data, there was no cluster containing 485 exclusively single-voxel regions.

486

487

------ Figure 5 and 6 here ------

488

489

Figure 7 illustrates all obtained clusters for c-partitions with low fuzzy separation $(c=8)$, without redundant clusters $(c=13)$, at high fuzzy separation $(c=18)$, and at the optimal c value that maximised $C V_{\text {new }}(\mathrm{c}=24)$. Identified voxels within the auditory cortex (i.e. voxels of interest) are

492 shown in the first axial slice of each c-partition. Voxels in the auditory cortex were grouped with 493 those in the occipital lobe at small $c$ values $(\mathrm{c}=8)$, but they became clearly segregated at larger $c$ 494 values (e.g. $c=18$ and $c=24$ ). Interestingly, identified voxels within the auditory cortex in the 495 c-partition with 24 clusters were remarkably similar to those identified with model-based SPM 496 methods (e.g. SPM map at p<0.05 FWE-corrected, Figure 8). Last but not least, the centroid of 497 the relevant cluster with activations in auditory regions (Cluster "1" of the 24-partitions in Figure 498 7) was strongly correlated with the experimental block design $(r=0.7, p<0.001)$. 
499

500

------ Figures 7 and 8 here ------

501

502 


\section{Discussion}

504 Using both simulated and real fMRI data, this study explored the usefulness of CV indices in

505 identifying the best c-partition with FCM. This study also examined the behaviour of different

506 compactness and separation measures, defined here as building blocks of the different $\mathrm{CV}$

507 indices. The optimal number of clusters varied with different $\mathrm{CV}$ indices, given that measures of

508 compactness and separation were influenced by different features of the fMRI data (e.g. the

509 expected high number of clusters, noise, and the amount of artefacts). By examining their

510 behaviour, a new $\mathrm{CV}$ index ( $C V_{\text {new }}$ ) was introduced, and this new index showed relatively good

511 robustness when clustering noisy data with high number of classes. Our study also highlighted

512 the importance of analysing different measures of separation and compactness in order to get a

513 better understating of the complex structure of the data.

514 The typical low signal-to-noise ratio in fMRI might be the most challenging issue that can hinder

515 the success of clustering techniques. Here, simulated data were based on Gaussian-like noise

516 distributions, and the success of different $\mathrm{CV}$ indices depended on the level of noise in the data.

517 Our findings are in line with previous studies that compared several CV indices on different

518 simulated datasets and found that $\mathrm{CV}$ indices may fail to indicate the true number of clusters in

519 noisy data that have high number of classes (Suleman 2017; Wang \& Zhang 2007; Zhou et al.

520 2014a). It might be the case their effectiveness might even be lesser given the complex nature of

521 noise in MRI images with significant correlations between voxels (Gudbjartsson \& Patz 1995;

522 Parrish et al. 2000). To ensure better data input to FCM, it is thus recommended to use different

523 pre-processing techniques that can reduce the impact of noise and improve data quality

524 (Caballero-Gaudes \& Reynolds 2017). The usefulness of such techniques with FCM on fMRI

525 data warrants further studies.

526 Perhaps more importantly, the results stressed the importance of reading the behaviour of

527 different separation and compactness measures, defined here as building blocks of CV indices, in

528 order to depict an accurate description of the fMRI data (cf. Figure 5). This is because it is most

529 likely that there are different meaningful c-partitions depending on the scale at which the

530 different clusters (i.e. brain networks) are segregated. Accordingly, it is not always useful to bias

531 the analysis towards one elusive single c-partition, but rather to appreciate that fMRI data might

532 encompass different plausible patterns or networks at different spatio-temporal scales (Orban et

533 al. 2015). Put another way, users need to relax the assumption that $\mathrm{c}_{\mathrm{opt}}$ must be unique, and look 
534 instead for complementary explanations of the data at different $\mathrm{c}_{\mathrm{opt}}$ values. For instance, using 535 fuzzy clustering on resting-state fMRI data, Lee and colleagues (Lee et al. 2012) identified two

536 optimal c-partitions with seven and eleven clusters that minimised a cluster dispersion measure 537 (used as a CV index). Interestingly, the c-partition with 11 clusters further subdivided some of 538 the clusters identified in the c-partition with seven clusters (Lee et al. 2012), most probably due 539 to the known hierarchical organization of the brain networks. Our results of the clustering of real 540 fMRI data also showed similar trends with clusters being further segregated with increasing 541 number of expected clusters (e.g. compare clusters with $\mathrm{c}=8$ to clusters with $\mathrm{c}=18$ in Figure 7).

542 Previous work suggested that, when $\mathrm{CV}$ indices fail to agree on the true number of clusters for

543 high-dimensional datasets, a combination of different indices into a single index should be 544 considered (Sheng et al. 2005; Zhou et al. 2014a). Specifically, by using a weighted sum of 545 several normalized CV indices, it has been shown that this weighted sum can improve the 546 confidence of clustering solutions. Ultimately, this approach aims to force an agreement between $547 \mathrm{CV}$ indices so that one optimal single c-partition is selected. However, this approach may not be 548 applicable to all contexts because (i) the number and types of $\mathrm{CV}$ indices to be combined are 549 arbitrary, (ii) there is no objective procedure to set optimal weights, and previous empirical work 550 showed that such weights are data-dependent (Zhou et al. 2014a), (iii) the weighted sum does not 551 properly deal with redundant information, given that $\mathrm{CV}$ indices are likely to share similar 552 compactness or separation measures, (iv) the relationships of some $\mathrm{CV}$ indices with the number 553 of expected clusters can take any arbitrary shape (e.g. Figure 5B), hence linear combinations 554 may not be suitable, and (v) this approach implicitly assumes that there must be one unique 555 'true' explanation of the data. Here I argue that summation of different CV indices might not be 556 useful for fMRI data clustering, because it ignores the possibility that different plausible 557 explanations (different c-partitions) exist for the same data. Differences between CV indices 558 should not be overlooked because they tend to highlight different existing features in the data. 559 The existence of different plausible explanations (c-partitions) of the same fMRI data can be 560 further illustrated when examining the different compactness and separation measures used in the 561 definition of the new CV index. More specifically, as illustrated in Figure 5B, $C V_{\text {new }}$ went 562 through three different phases: (i) low values for $\mathrm{c}<15$, (ii) a plateau with high optimal values for $56315<\mathrm{c}<28$, and (iii) another plateau for $\mathrm{c}>28$. The three phases indicated different segregated data 564 structures depending on the predominance of either compactness or separation measures (Figure 
565 5A). For example, high fuzzy separation with well-isolated clusters was only achieved at c $>15$,

566 as reflected in the behaviour of $K_{m}$ and $I D_{\text {int } r a}$; however, when c increased the c-partitions

567 became less compact (see $F C$ ), with higher fuzzy overlap and over-classification when c

568 increased beyond 28 clusters (see $I D_{\text {inter }}$ ). Given the expected small proportion of task-related

569 activations in the auditory cortex, a segregation of relevant auditory voxels was only achieved

570 with $\mathrm{c}>15$ clusters, for a similar rationale see (Chuang et al. 1999). In sum, looking at different

571 compactness and separation measures, in addition to $C V_{n e w}$ index, can provide a richer

572 representation of the clustering results so that users can select the most useful c-partition among 573 many potential possibilities.

574 Other methodological issues warrant further investigations. For instance, it might be interesting

575 to test these CV indices with other varieties of FCM algorithms that incorporated spatial

576 constraints during the minimisation of the objective function $J_{m}$ (e.g. (Ahmed et al. 2002; Liew et

577 al. 2000)), which can take into account the inherent spatial dependencies between neighbouring

578 voxels (e.g. dependencies inflated by the spatial resampling and smoothing in fMRI). This would

579 for instance penalise implausible solutions (c-partitions) with isolated voxels (e.g. Figure 6). In

580 addition, if outlier voxels existed in a dataset, this would artificially yield optimal CV values for

581 c-partitions with a small number of clusters. In this context, it is useful to combine these CV

582 indices with robust clustering techniques (for a review see (Dave \& Krishnapuram 1997)),

583 adaptive distance measures (Tang et al. 2015), or other modified fuzzy clustering algorithms

584 (e.g. (Dik et al. 2014; Kao \& Huang 2013; Keller 2000; Seghier et al. 2007)). Another

585 challenging issue is to give meaning to the different identified clusters. Typically, users have to

586 set objective criteria to distinguish relevant clusters from noise or artefact-driven clusters. For

587 instance, for task-related fMRI data, clusters of interest are expected to have centroids similar

588 (highly correlated) to the paradigm (Chuang et al. 1999; Fadili et al. 2000; Goutte et al. 1999;

589 Jahanian et al. 2005), as illustrated in Figure 7. For task-free fMRI data, irrelevant clusters

590 should be discarded, including clusters that are less consistent across sessions (Levin \& Uftring

591 2001) or when they include irrelevant brain voxels (e.g. in the white matter, ventricles,

592 cerebrospinal fluid, arteries) (Ma et al. 2011).

593 Although FCM can provide useful data-driven explanations, deciding which clustering method is

594 best for fMRI data remains an open question (Derntl \& Plant 2016). Typically, selecting a

595 specific clustering algorithm entails a trade-off between different criteria (e.g. accuracy versus 
596 stability (Thirion et al. 2014)), with different methods may yield different clustering solutions.

597 Many previous fMRI studies for instance have compared FCM against other data-driven

598 methods, but findings varied considerably across studies, probably due to differences in fMRI

599 data features in particular in terms of contrast-to-noise ratio and the level of physiological noise

600 (Baumgartner et al. 2000; Dimitriadou et al. 2004; Lange et al. 2006; Wismuller et al. 2004).

601 One popular data-driven method in the current literature is independent component analysis

602 (ICA). ICA allows the detection of unexpected brain responses to stimuli, dissociation of

603 functional networks and can be used as a powerful denoising tool (Stone 2002). Previous work

604 (Meyer-Baese et al. 2004; Smolders et al. 2007) have shown that FCM may outperform ICA

605 when analyzing task-related fMRI data with good contrast-to-noise ratio. Nonetheless, it is fair to

606 say that any comparison between ICA and FCM is an empirical question that is contingent on the

607 nature of the fMRI data, the exact parametrization of FCM (Schwämmle \& Jensen 2010), the

608 type of ICA algorithm, and the number of independent components (McKeown et al. 2003).

609

610 Conclusions

611 Unsupervised FCM with different CV indices is a useful way of analysing model-free

$612 \mathrm{fMRI}$ datasets, an alternative to the widely used independent component analysis methods. It is

613 recommended to combine different $\mathrm{CV}$ indices in order to draw a complete picture of the

614 structure of the date and to make sure that no relevant or meaningful clusters are missed. The

615 assumption here is that different $\mathrm{CV}$ indices may point to different optimal c-partitions, given the

616 heterogeneous behaviour of many measures of compactness and separation. Rather than

617 discarding discrepancies between $\mathrm{CV}$ indices, such discrepancies should be appreciated because

618 they reflect the hierarchical organization of brain networks. This was clearly visible for instance

619 when analysing the different phases of the plot of the new CV index against the number of

620 clusters. Overall, the existence of different c-partitions for the same fMRI data should not be

621 overlooked in future clustering studies.

622

623 
624 References

625

626

627

628

629

630

631

632

633

634

635

636

637

638

639

640

641

642

643

644

645

646

647

648

649

650

651

652

653

654

655

656

657

658

659

660

661

662

663

664

665

666

667

668

669

Ahmed MN, Yamany SM, Mohamed N, Farag AA, and Moriarty T. 2002. A modified fuzzy cmean algorithm for bias field estimation and segmentation of MRI data. IEEE Trans Med Imaging 21:193-199.

Alexiuk MD, and Pizzi NJ. 2004. Cluster validation indices for fMRI data: Fuzzy C-Means with feature partitions versus cluster merging strategies. Fuzzy Information, 2004 Processing NAFIPS '04. Alberta, Canada.

Aljobouri HK, Jaber HA, Koçak OM, Algin O, and Çankaya I. 2018. Clustering fMRI data with a robust unsupervised learning algorithm for neuroscience data mining. J Neurosci Methods 299:45-54.

Bandettini PA, Jesmanowicz A, Wong EC, and Hyde JS. 1993. Processing strategies for timecourse data sets in functional MRI of the human brain. Magnetic Resonance in Medecine 30:161-173.

Bartels A, and Zeki S. 2004. Functional brain mapping during free viewing of natural scenes. Hum Brain Mapp 21:75-85.

Bartels A, and Zeki S. 2005. The chronoarchitecture of the cerebral cortex. Philos Trans R Soc Lond B Biol Sci 360:733-750.

Baumgartner R, Ryner L, Richter W, Summers R, Jarmasz M, and Somorjai R. 2000. Comparison of two exploratory data analysis methods for fMRI: fuzzy clustering vs. principal component analysis. Magn Reson Imaging 18:89-94.

Baumgartner R, Windischberger C, and Moser E. 1998. Quantification in functional magnetic resonance imaging: Fuzzy clustering vs. correlation analysis. Magnetic Resonance Imaging 16:115-125.

Bensaid AM, Hall LO, Bezdek JC, Clarke LP, Silbiger ML, Arrington JA, and Murtaqh RF. 1996. Validity-guided (re)clustering with applications to imagesegmentation. IEEE Trans Fuzzy Sys 4:112-123.

Bezdek JC. 1981. Pattern recognition with fuzzy objective functions algorithms. New York: Plenum Press.

Bezdek JC, Hall LO, Clark MC, Goldgof DB, and Clarke LP. 1997. Medical image analysis with fuzzy models. Stat Methods Med Res 6:191-214.

Bezdek JC, and Pal NR. 1998. Some new indexes of cluster validity. IEEE Trans Sys Man Cyber B 28:301-315.

Bouguessa M, Wang S, and Sun H. 2006. An objective approach to cluster validation. Pattern Recogn Lett 27:1419-1430.

Caballero-Gaudes C, and Reynolds RC. 2017. Methods for cleaning the BOLD fMRI signal. Neuroimage (in press).

Chuang KH, Chiu MJ, Lin CC, and Chen JH. 1999. Model-free functional MRI analysis using kohonen clustering neural network and fuzzy c-means. IEEE Trans Med Imaging 18:11171128.

Dave RN. 1996. Validating fuzzy partition obtained through c-shells clustering. Pattern Recogn Lett 17:613-623.

Dave RN, and Krishnapuram R. 1997. Robust clustering methods: a united view. IEEE Trans Fuzzy Sys 5:270-293.

Derntl A, and Plant C. 2016. Clustering techniques for neuroimaging applications. WIREs Data Mining Knowl Discov 6:22-36. 
670 Dik A, Jebari K, Bouroumi A, and Ettouhami A. 2014. A New Fuzzy Clustering by Outliers.

671

672

673

674

675

676

677

678

679

680

681

682

683

684

685

686

687

688

689

690

691

692

693

694

695

696

697

698

699

700

701

702

703

704

705

706

707

708

709

710

711

712

713 Journal of Engineering and Applied Sciences 9:372-377.

Dimitriadou E, Barth M, Windischberger C, Hornik K, and Moser E. 2004. A quantitative comparison of functional MRI cluster analysis. Artif Intell Med 31:57-71.

DonGiovanni D, and Vaina LM. 2016. Select and Cluster: A Method for Finding Functional Networks of Clustered Voxels in fMRI. Computational Intelligence and Neuroscience 2016:4705162.

Dubes RC. 1987. How many clusters are best? an experiment. Pattern Recogn 20:645-663.

Esposito F, Formisano E, Seifritz E, Goebel R, Morrone R, Tedeschi G, and di Salle F. 2002. Spatial independent component analysis of functional MRI time-series: to what extent do results depend on the algorithm used? Hum Brain Mapp 16:146-157.

Fadili MJ, Ruan S, Bloyet D, and Mazoyer B. 2000. A multistep unsupervised fuzzy clustering analysis of fMRI time series. Hum Brain Mapp 10:160-178.

Fadili MJ, Ruan S, Bloyet D, and Mazoyer B. 2001. On the number of clusters and the fuzziness index for unsupervised FCA application to BOLD fMRI time series. Med Image Anal 5:5567.

Fatemizadeh E, Taalimi A, and Davoudi H. 2009. Extracting activated regions of fMRI data using unsupervised learning. IEEE - INNS - ENNS International Joint Conference on Neural Networks. Atlanta, Ga, USA. p 641-645.

Fukuyama Y, and Sugeno M. 1989. A new method for choosing the number of clusters for fuzzy c-means method. 5th Fuzzy System Symposium. p 247-250.

Gath I, and Geva AB. 1989. Unsupervised optimal fuzzy clustering. IEEE Trans Pattern Anal Mach Intel 11:773-781.

Geva AB, Steinberg Y, Bruckmair S, and Nahum G. 2000. A comparison of cluster validity criteria for a mixture of normal distributed data. Pattern Recogn Lett 21:511-529.

Golay X, Kollias S, Stoll G, Meier D, Valavanis A, and Boesiger P. 1998. A new correlation-based fuzzy logic clustering algorithm for fMRI. Magn Reson Med 40:249-260.

Goutte C, Toft P, Rostrup E, Nielsen FA, and Hansen LK. 1999. On clustering fMRI time series. Neuroimage 9:298-310.

Gu J, Cao Z, Zheng X, and Aihua C. 2005. Treatment of ill-balanced dataasets of fMRI with modified fuzzy c-means method. 27th Ann Confer IEEE Eng Med Biol. Shanghai China. p 1411-1414.

Gudbjartsson H, and Patz S. 1995. The Rician distribution of noisy MRI data. Magn Reson Med 34:910-914.

Hammah RE, and Curran JH. 2000. Validity measures for the fuzzy cluster analysis of orientations. IEEE Trans Pattern Anal Mach Intel 22:1467-1472.

Hasson U, Nir Y, Levy I, Fuhrmann G, and Malach R. 2004. Intersubject synchronization of cortical activity during natural vision. Science 303:1634-1640.

He H, Tan Y, and Fujimoto K. 2016. Estimation of optimal cluster number for fuzzy clustering with combined fuzzy entropy index. 2016 IEEE International Conference on Fuzzy Systems (FUZZ-IEEE). Vancouver, BC, Canada.

Hu Y, Zuo C, Yang Y, and Qu F. 2011. A cluster validity index for fuzzy c-means clustering. 2011 International Conference on System Science, Engineering Design and Manufacturing Informatization (ICSEM). Guiyang, China. 
714

715

716

717

718

719

720

721

722

723

724

725

726

727

728

729

730

731

732

733

734

735

736

737

738

739

740

741

742

743

744

745

746

747

748

749

750

751

752

753

754

755

756

757

758

Jahanian H, Hossein-Zadeh GA, Soltanian-Zadeh H, and Ardekani BA. 2004. Controlling the false positive rate in fuzzy clustering using randomization: application to fMRI activation detection. Magn Reson Imaging 22:631-638.

Jahanian H, Soltanian-Zadeh H, and Hossein-Zadeh GA. 2005. Functional magnetic resonance imaging activation detection: Fuzzy cluster analysis in wavelet and multiwavelet domains. J Magn Reson Imaging 22:381-389.

Kao LJ, and Huang YP. 2013. A Novel Fuzzy Clustering Method with No Outliers Influence. Applied Mechanics and Materials 300-301:735-739.

Keller A. 2000. Fuzzy clustering with outliers. 19th International Conference of the North American Fuzzy Information Processing Society (NAFIPS) Atlanta, GA, USA.

Kim DJ, Park YW, and Park DJ. 2001. A Novel validity index for determination of the optimal number of clusters. IEICE Trans Inf Syst 84-D:281-285.

Kim DW, Lee KH, and Lee D. 2003. Fuzzy cluster validation index based on inter-cluster proximity. Pettern Recogn Lett 24:2561-2574.

Kim M, and Ramakrishna RS. 2005. New indices for cluster validity assessment. Pattern Recogn Lett 26:2353-2363.

Krishnapuram R, and Keller JM. 1993. A possibilistic approach to clustering. IEEE Trans Fuzzy Sys 1:98-110.

Kwon SH. 1998. Cluster validity index for fuzzy clustering. Electron Lett 34:2176-2177.

Lange O, Meyer-Baese A, Hurdal M, and Foo S. 2006. A comparison between neural and fuzzy cluster analysis techniques for functional MRI. Biomedical Signal Process Control 1:243252.

Lange O, Meyer-Baese A, Wismueller A, Hurdal M, Sumners D, and Auer D. 2004. Model-free functional MRI analysis using improved fuzzy cluster analysis techniques. In: Priddy KL, editor. SPIE Intelligent Computing: Theory and Applications. p 19-28.

Lee MH, Hacker CD, Snyder AZ, Corbetta M, Zhang D, Leuthardt EC, and Shimony JS. 2012. Clustering of Resting State Networks. PLoS One 7:e40370.

Levin DN, and Uftring SJ. 2001. Detecting brain activation in fMRI data without prior knowledge of mental event timing. Neuroimage 13:153-160.

Liew AWC, Leung SH, and Lau WH. 2000. Fuzzy image clustering incorporating spatial continuity. IEE Proc Vision Image Signal Process 147:185-192.

Lin PL, Huang PW, Wu CH, and Huang SM. 2016. An efficient validity index method for datasets with complex-shaped clusters. 2016 International Conference on Machine Learning and Cybernetics (ICMLC). Jeju, South Korea.

Lu Y, Jiang T, and Zang Y. 2004. A split-merge-based region-growing method for fMRI activation detection. Hum Brain Mapp 22:271-279.

Ma S, Correa NM, Li XL, Eichele T, Calhoun VD, and Adalı T. 2011. Automatic identification of functional clusters in FMRI data using spatial dependence. IEEE Trans Biomed Eng 58:3406-3417.

Malinen S, Hlushchuk Y, and Hari R. 2007. Towards natural stimulation in fMRI-Issues of data analysis. Neuroimage 35:131-139.

Maulik U, and Bandyopadhyay S. 2002. Performance evaluation of some clustering algorithms and validity indices. IEEE Trans Pattern Anal Mach Intel 24:1650-1654.

McKeown MJ, Hansen LK, and Sejnowsk TJ. 2003. Independent component analysis of functional MRI: what is signal and what is noise? Curr Opin Neurobiol 13:620-629. 
759

760

761

762

763

764

765

766

767

768

769

770

771

772

773

774

775

776

777

778

779

780

781

782

783

784

785

786

787

788

789

790

791

792

793

794

795

796

797

798

799

800

801

802
McKeown MJ, Makeig S, Brown GG, Jung TP, Kindermann SS, Bell AJ, and Sejnowski TJ. 1998. Analysis of fMRI data by blind separation into independent spatial components. Hum Brain Mapp 6:160-188.

Meyer-Baese A, Wismueller A, and Lange O. 2004. Comparison of two exploratory data analysis methods for fMRI: unsupervised clustering versus independent component analysis. IEEE Trans Inf Technol Biomed 8:387-398.

Moller U, Ligges M, Georgiewa P, Grunling C, Kaiser WA, Witte H, and Blanz B. 2002. How to avoid spurious cluster validation? A methodological investigation on simulated and fMRI data. Neuroimage 17:431-446.

Orban P, Doyon J, Petrides M, Mennes M, Hoge R, and Bellec P. 2015. The Richness of TaskEvoked Hemodynamic Responses Defines a Pseudohierarchy of Functionally Meaningful Brain Networks. Cereb Cortex 25:2658-2669.

Pakhira MK, Bandyopadhyay S, and Maulik U. 2004. Validity index for crisp and fuzzy clusters. Pattern Recogn 37:487-501.

Pakhira MK, Bandyopadhyay S, and Maulik U. 2005. A study of some fuzzy cluster validity indices, genetic clustering and application to pixel classification. Fuzzy Sets Sys 155:191214.

Pal NR, and Bezdek JC. 1995. On cluster validity for the fuzzy c-means model. IEEE Trans Fuzzy Sys 3:370-379.

Parrish TB, Gitelman DR, LaBar KS, and Mesulam MM. 2000. Impact to signal-to-noise on Functional MRI. Magnetic Resonance in Medecine 44:925-932.

Pena JM, Lozano JA, and Larranaga P. 1999. An empirical comparison of four initialization methods for the K-Means algorithm. Pattern Recogn Lett 20:1027-1040.

Quiqley MA, Haughton VM, Carew J, Cordes D, Moritz CH, and Meyerand ME. 2002. Comparison of independent component analysis and conventional hypothesis-driven analysis for clinical functional MR image processing. Am J Neuroradiol 23:49-58.

Ren M, Liu P, Wang Z, and Yi J. 2016. A Self-Adaptive Fuzzy c-Means Algorithm for Determining the Optimal Number of Clusters. Comput Intell Neurosci 2016:2647389.

Rezaee B. 2010. A cluster validity index for fuzzy clustering. Fuzzy Sets Syst 161:3014-3025.

Rezaee MR, Lelieveldt BPF, and Reider JHC. 1998. A new cluster validity index for the fuzzy cmean. Pattern Recogn Lett 19:237-246.

Rhee HS, and Oh KW. 1996. A performance measure for the fuzzy cluster validity. IEEE:364369.

Schwämmle V, and Jensen ON. 2010. A simple and fast method to determine the parameters for fuzzy c-means cluster analysis. Bioinformatics 56:1841-2848.

Seghier ML, Friston KJ, and Price CJ. 2007. Detecting subject-specific activations using fuzzy clustering. Neuroimage 36:594-605.

Seghier ML, and Price CJ. 2009. Dissociating functional brain networks by decoding the betweensubject variability. Neuroimage 45:349-359.

Selim AZ, and Ismail MA. 1986. On the local optimality of the Fuzzy ISODATA clustering algorithm. IEEE Trans Pattern Anal Mach Intel 8:284-288.

Sheng W, Swift S, Zhang L, and Liu X. 2005. A weighted sum validity function for clustering with a hybrid niching genetic algorithm. IEEE Trans Syst Man Cyber (Part B) 35:11561167. 
803

804

805

806

807

808

809

810

811

812

813

814

815

816

817

818

819

820

821

822

823

824

825

826

827

828

829

830

831

832

833

834

835

836

837

838

839

840

841

842

843

844

845

846

847

848

Smolders A, De Martino F, Staeren N, Scheunders P, Sijbers J, Goebel R, and Formisano E. 2007. Dissecting cognitive stages with time-resolved fMRI data: a comparison of fuzzy clustering and independent component analysis. Magn Reson Imaging 25:860-868.

Soille P. 2003. Morphological Image Analysis: Principles and Applications. New York: SpringerVerlag.

Soltanian-Zadeh H, Peck DJ, Hearshen DO, and Lajiness-O'Neill RR. 2004. Model-independent method for fMRI analysis. IEEE Trans Med Imaging 23:285-296.

Stone JV. 2002. Independent component analysis: an introduction. Trends Cogn Sci 6:59-64.

Suleman A. 2017. Measuring the congruence of fuzzy partitions in fuzzy c-means clustering. Applied Soft Computing 52:1285-1295.

Sun H, Wang S, and Jiang Q. 2004. FCM-based model selection algorithms for determining the number of clusters. Pattern Recogn 37:2027-2037.

Tang X, Zeng W, Wang N, and Yang J. 2015. An adaptive RV measure based fuzzy weighting subspace clustering (ARV-FWSC) for fMRI data analysis. Biomedical Signal Process Control 22:146-154.

Thirion B, Varoquaux G, Dohmatob E, and Poline JB. 2014. Which fMRI clustering gives good brain parcellations? Front Neurosci 8:167.

Tibshirani R, Walther G, and Hastie T. 2001. Estimating the number of clusters in a data set via the gap statistic. $J R$ Statist $S o c$ B 63:411-423.

Tsekouras GE, and Sarimveis H. 2004. A new approach for measuring the validity of the fuzzy cmeans algorithm. Adv Eng Software 35:567-575.

Wang W, and Zhang Y. 2007. On fuzzy cluster validity indices. Fuzzy Sets Sys 158:2095-2117.

Windham MP. 1981. Cluster validity for fuzzy clustering algorithms. J Fuzzy Sets Syst 5.

Windischberger C, Barth M, Lamm C, Schroeder L, Bauer H, Gur RC, and Moser E. 2003. Fuzzy cluster analysis of high-field functional MRI data. Artif Intell Med 29:203-223.

Wismuller A, Meyer-Base A, Lange O, Auer D, Reiser MF, and Sumners D. 2004. Model-free functional MRI analysis based on unsupervised clustering. J Biomed Informatics 37:10-18.

$\mathrm{Wu}$ KL, and Yang MS. 2005. A cluster validity index for fuzzy clustering. Pattern Recogn Lett 26:1275-1291.

Xie XL, and Beni G. 1991. A validity measure for fuzzy clustering. IEEE Trans Pattern Anal Mach Intel 13:841-847.

Yang SL, Li K, Liang Z, Li W, and Xue Y. 2018. A novel cluster validity index for fuzzy C-means algorithm. Soft Computing 22:1921-1931.

Yu J, Cheng Q, and Huang H. 2004. Analysis of the Weighting Exponent in the FCM. IEEE Trans Sys Man Cyber B 34:634-639.

Yu J, and Li CX. 2006. Novel cluster validity index for FCM algorithm. J Comput Sci Technol 21:137-140.

Zacks JM, Braver TS, Sheridan MA, Donaldson DI, Snyder AZ, Ollinger JM, Buckner RL, and Raichle ME. 2001. Human brain activity time-locked to perceptual event boundaries. Nat Neurosci 4:651-655.

Zahid N, Aboulala O, Limouri M, and Essaid A. 1999a. Unsupervised fuzzy clustering. Pattern Recogn Lett 20:123-129.

Zahid N, Limouri M, and Essaid A. 1999b. A new cluster-validity for fuzzy clustering. Pettern Recogn 32:1089-1097.

Zhang D, Ji M, Yang J, Zhang Y, and Xie F. 2014. A novel cluster validity index for fuzzy clustering based on bipartite modularity. Fuzzy Sets Syst 253:122-137. 
849 Zhou K, Ding S, Fu C, and Yang SL. 2014a. Comparison and Weighted Summation Type of Fuzzy $850 \quad$ Cluster Validity Indices. Int J Comput Commun 9:370-378.

851 Zhou K, Fu C, and Yang SL. 2014b. Fuzziness parameter selection in fuzzy c-means: The 852 perspective of cluster validation. Science China 57:112206.

853 
855 Legends

856 Figure 1: illustrates the behaviour of different measures of compactness and separation: FCM on the 1-cluster (A) and the n-cluster (B) dataset. The number of expected clusters varied between 2 and 19. See full definition of the different measures in the Methods section.

859

860

Figure 2: illustrates the behaviour of different measures of compactness and separation during

861 FCM of the 7-cluster dataset with low $(A, \sigma=1)$ and high $(B, \sigma=4)$ noise levels. See full

862 definition of the different measures in the Methods section.

863

864 Figure 3: plots of the CV indices for the simulated 3-clusters (A), 7 clusters (B) and 11-clusters

865 (C) with low noise level $(\sigma=1)$, when number of expected clusters increased from 2 to 19. All CV indices successfully indicated the expected number of clusters $\left(\mathrm{c}_{\mathrm{opt}}=3\right.$ in $\mathrm{a}, \mathrm{c}_{\mathrm{opt}}=7$ 868

Figure 4: plots of the CV indices for the simulated 3-clusters (A), 7 clusters (B) and 11-clusters (C) with high noise levels $(\sigma=4)$, when the number of expected clusters increased from 2 to 19. Only the new CV index identified the correct 11-partition at this level of noise.

872 in $b$ and $c_{o p t}=11$ in $c$ ). See full definition of these indices in the Methods section.

Figure 5: illustrates the results using real fMRI data. (A) Different measures of compactness and separation and (B) the different $\mathrm{CV}$ indices. The number of expected clusters varied from 2 and 39.

Figure 6: (A) Regions' sizes (in number of voxels) for each crisp c-partition (at an arbitrary threshold of $U>0.5$ ). Each dot (diamond shape) represents the size of one region in any 
884 Figure 7: FCM results at different $\mathrm{c}$ values (A: $\mathrm{c}=8, \mathrm{~B}: \mathrm{c}=13, \mathrm{C}: \mathrm{c}=18$ and D: $\mathrm{c}=24)$. Each

885

886

887

888

889

890

891

892

893

894

895

896

897

898

899

900

901

902

903

904

905

906

907

908

obtained cluster (a 3D image) of each c-partition is illustrated by its most representative axial slice, with $U$ values varying from 0.1 to 1.0 . Cluster label is shown at the top-left corner of each axial slice (in white) and the MNI-z coordinate is indicated in black. For illustration purposes, the cluster that contained the expected activated voxels within the auditory cortex is labelled as Cluster ' 1 '. The scatter plot (E) illustrates the correlations between the centroids of the 24-partition and the experimental block design (y-axis) against the fuzzy cardinality (cf. Equation 6) of each cluster (x-axis). Only one cluster showed significant correlation $(\mathrm{p}<0.001)$ with the experimental design $(\mathrm{r}=0.7)$. The fuzzy cardinality was divided by the total number of voxels, which would approximately reflect the 'proportion' of voxels contained in each cluster (average proportion around $4 \%(=1 / \mathrm{c})$ ). Using the spatial location of the clustered voxels, one can potentially interpret the results of the FCM 24-partition (D). For example, Cluster 1 is showing auditory activations (cluster of interest) that highly correlated with the experimental block design $(r=0.7)$; Clusters 2-4 illustrate voxels in the visual system; Clusters 5-8 illustrate cerebellar and subcortical regions; Clusters 9-10 illustrate different medial parts of the default mode network; Clusters 11-12 contain voxels in ventral brain regions that are prone to MR signal loss; Clusters 13 and 14 are dominated by motion artefacts; Cluster 15 mainly shows CSF voxels; Clusters 19-24 contain white matter voxels. $\mathrm{L}=$ left hemisphere, $\mathrm{R}=$ right hemisphere.

Figure 8: SPM results illustrated with the function 'montage' of SPM12, with axial slices varying between $\mathrm{MNI}-\mathrm{z}=-16 \mathrm{~mm}$ to $\mathrm{MNI}-\mathrm{z}=+36 \mathrm{~mm}$. (A) results at a very liberal threshold of $\mathrm{p}<0.05$ uncorrected, (B) at $\mathrm{p}<0.05$ FWE-corrected. $\mathrm{L}=$ left hemisphere, $\mathrm{R}=$ right

$$
\text { hemisphere. }
$$




\section{Table 1 (on next page)}

List of the selected cluster validity (CV) indices. 
1

2 Table 1: List of the selected cluster validity (CV) indices.

3

\begin{tabular}{|l|l|l|l|}
\hline$C V$ index & Proposed by & range & ${\text { Value at } \mathrm{c}_{\mathrm{opt}}}^{\text {Rezaee-Lelieveldt-Reider index }}$ \\
\hline$C V_{R L R}$ & $\begin{array}{l}\text { (1998). A modified version was } \\
\text { used here (Sun et al., 2004). }\end{array}$ & {$[0,+\infty[$} & minimal \\
\hline$C V_{Z L E}$ & Zahid-Limouri-Essaid index (1999) & ]$-\infty,+\infty[$ & maximal \\
\hline$C V_{G V}$ & Geva index (2000). & {$[0,+\infty[$} & maximal \\
\hline$C V_{K P}$ & Kim-Park index (2001) & {$[0,+\infty[$} & minimal \\
\hline$C V_{P B M}$ & $\begin{array}{l}\text { Pakhira-Bandyopadhyay-Maulik } \\
\text { index (2004) }\end{array}$ & {$[0,+\infty[$} & maximal \\
\hline$C V_{W Y}$ & Wu-Yang index (2005) & {$[-\mathrm{c}, \mathrm{c}]$} & maximal \\
\hline$C V_{B W S}$ & Bouguessa-Wang-Sun index (2006) & {$[0,+\infty[$} & maximal \\
\hline$C V_{n e w}$ & A new CV index & {$[0,+\infty[$} & maximal \\
\hline & & & \\
\hline
\end{tabular}

4

5

6

7 


\section{Figure 1}

Illustration of the behaviour of different measures of compactness and separation.

FCM on the 1-cluster (A) and the n-cluster (B) dataset. The number of clusters varied

between 2 and 19. See full definition of the different measures in the Methods section. 
A
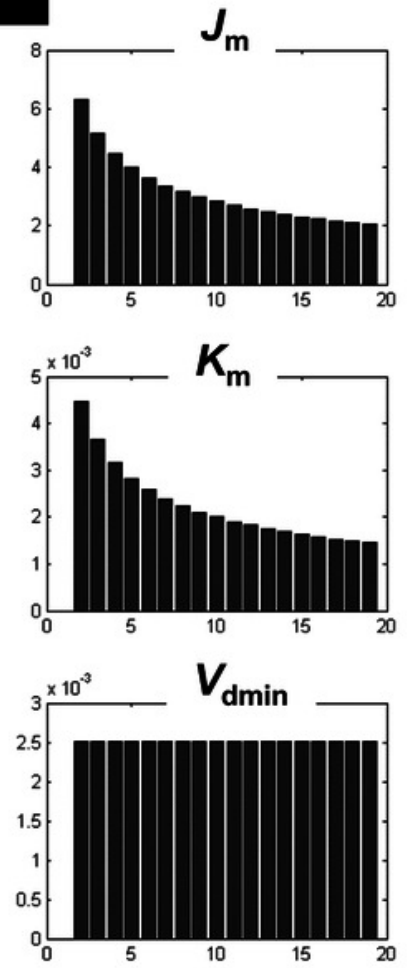

\section{B}
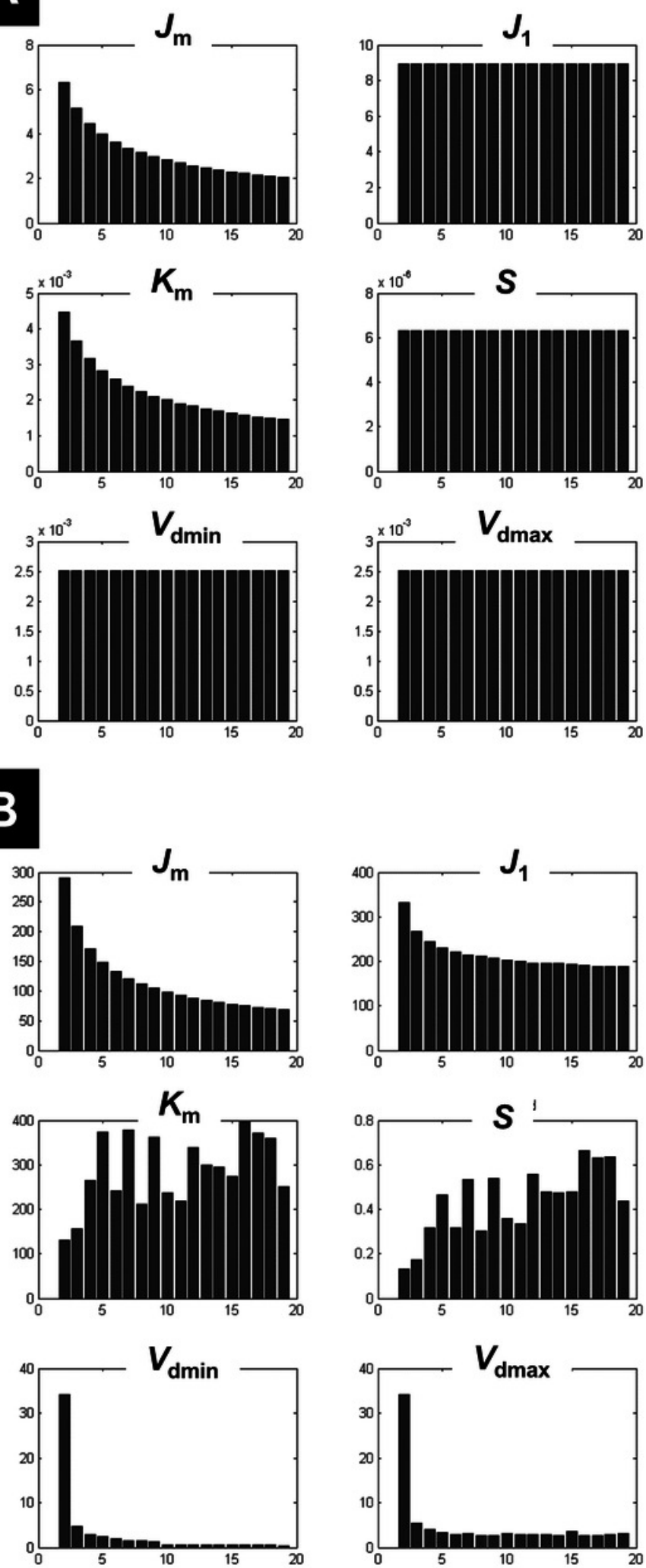
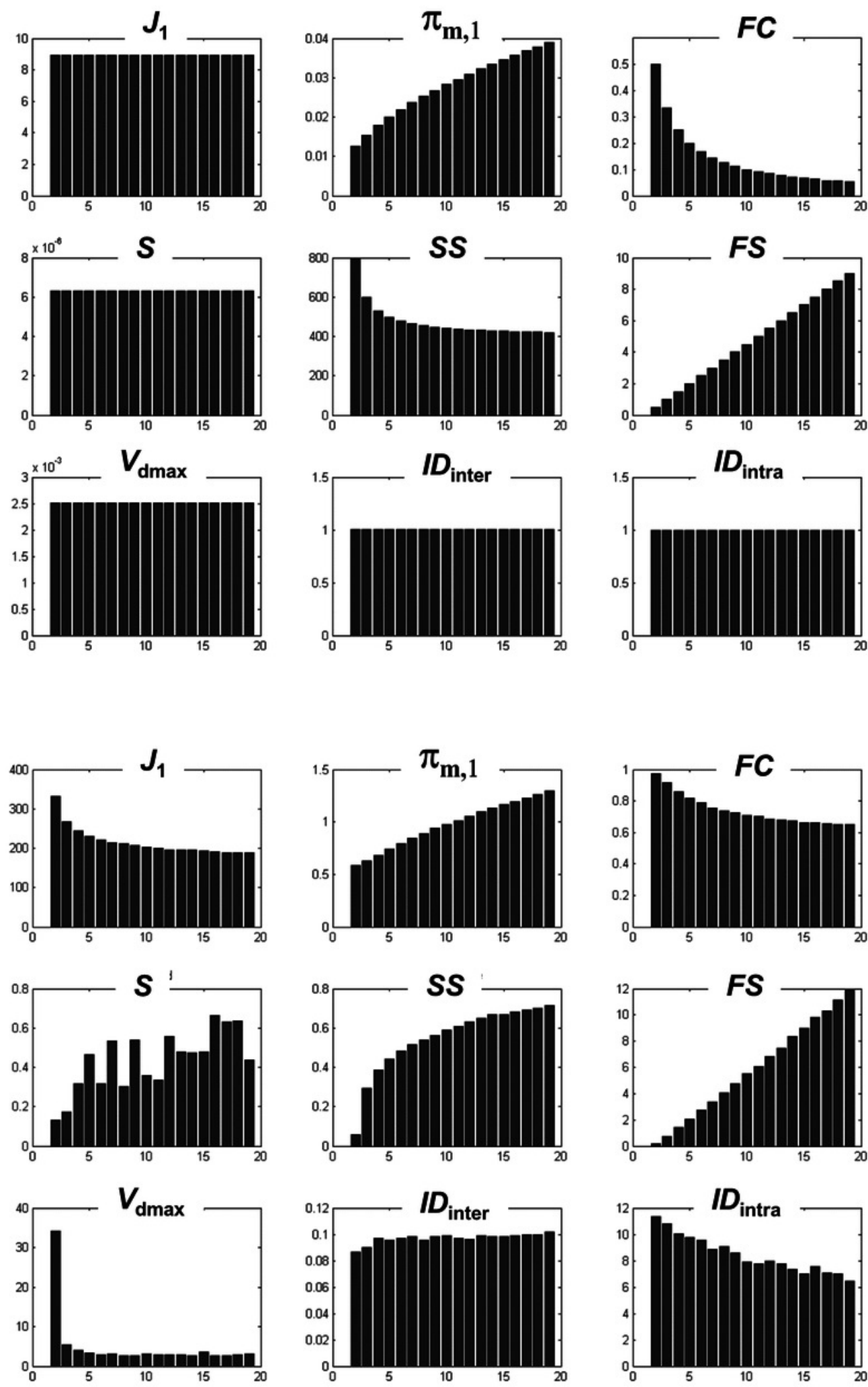
Figure 2

Illustration of the behaviour of different measures of compactness and separation at different noise levels.

The behaviour of different measures of compactness and separation during FCM of the 7cluster dataset with low $(A, \sigma=1)$ and high $(B, \sigma=4)$ noise levels. See full definition of the different measures in the Methods section. 
A
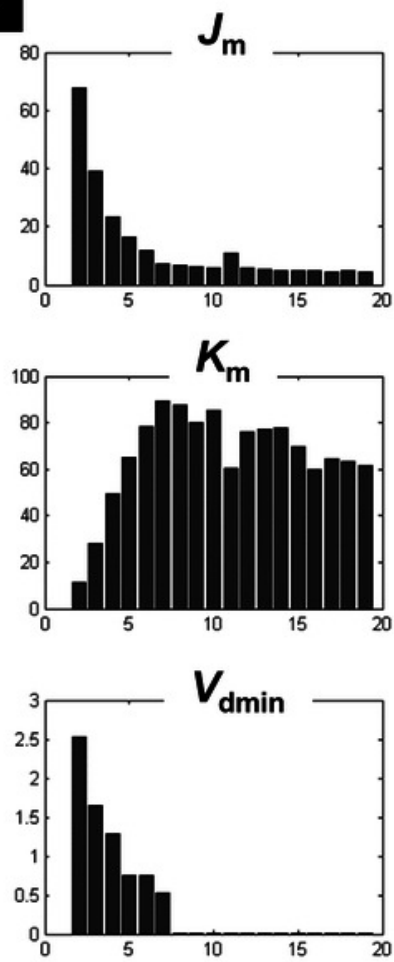

\section{B}
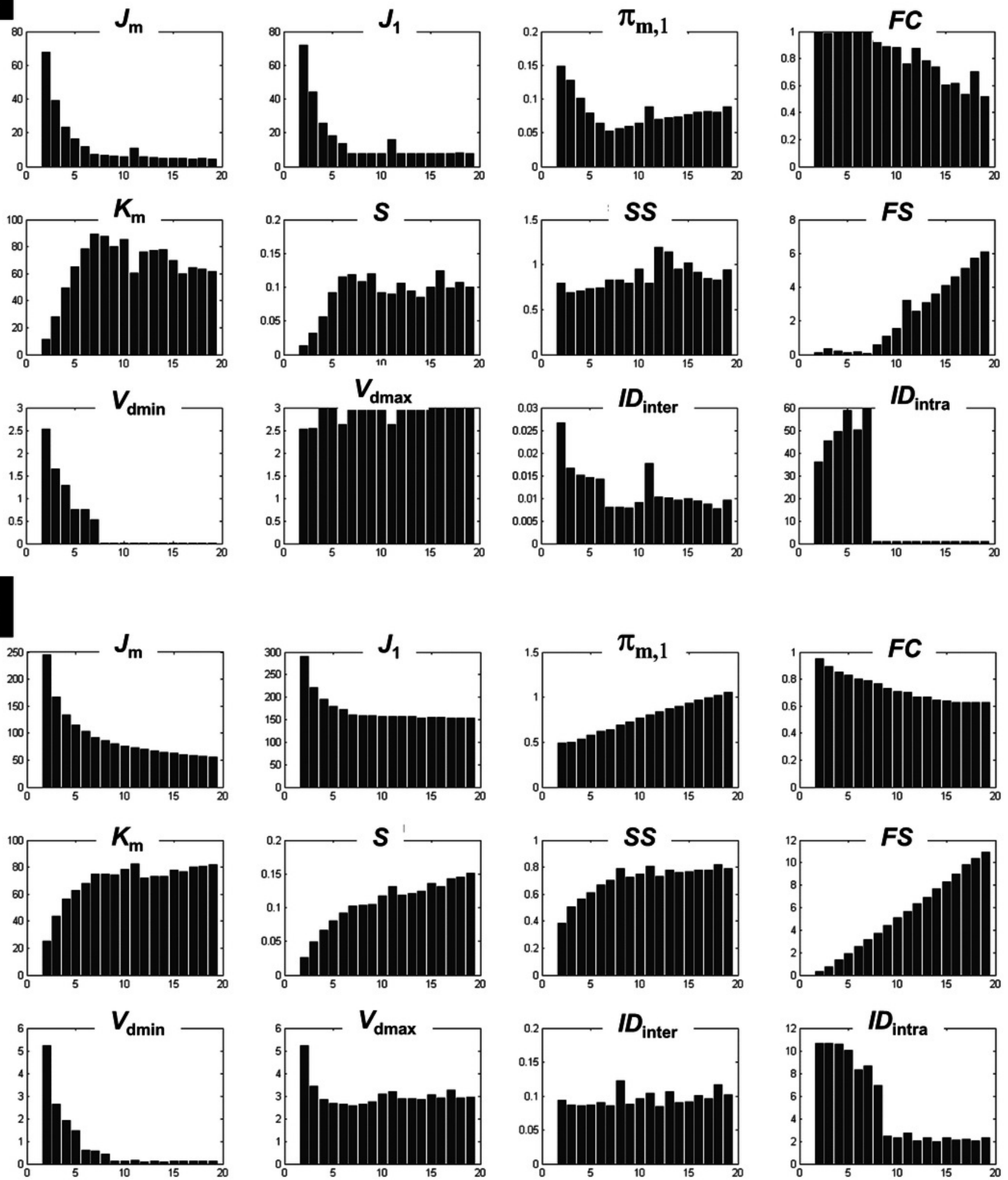
Figure 3

Plots of the CV indices for the simulated data at low noise level.

Plots of the CV indices for the simulated 3-clusters (A), 7 clusters (B) and 11-clusters (C) with low noise level $(\sigma=1)$, when number of clusters increased from 2 to 19. All CV indices successfully indicated the expected number of clusters ( $c_{\text {opt }}=3$ in a, $c_{\text {opt }}=7$ in $b$ and $c_{\text {opt }}=$ 11 in c). See full definition of these indices in the Methods section. 

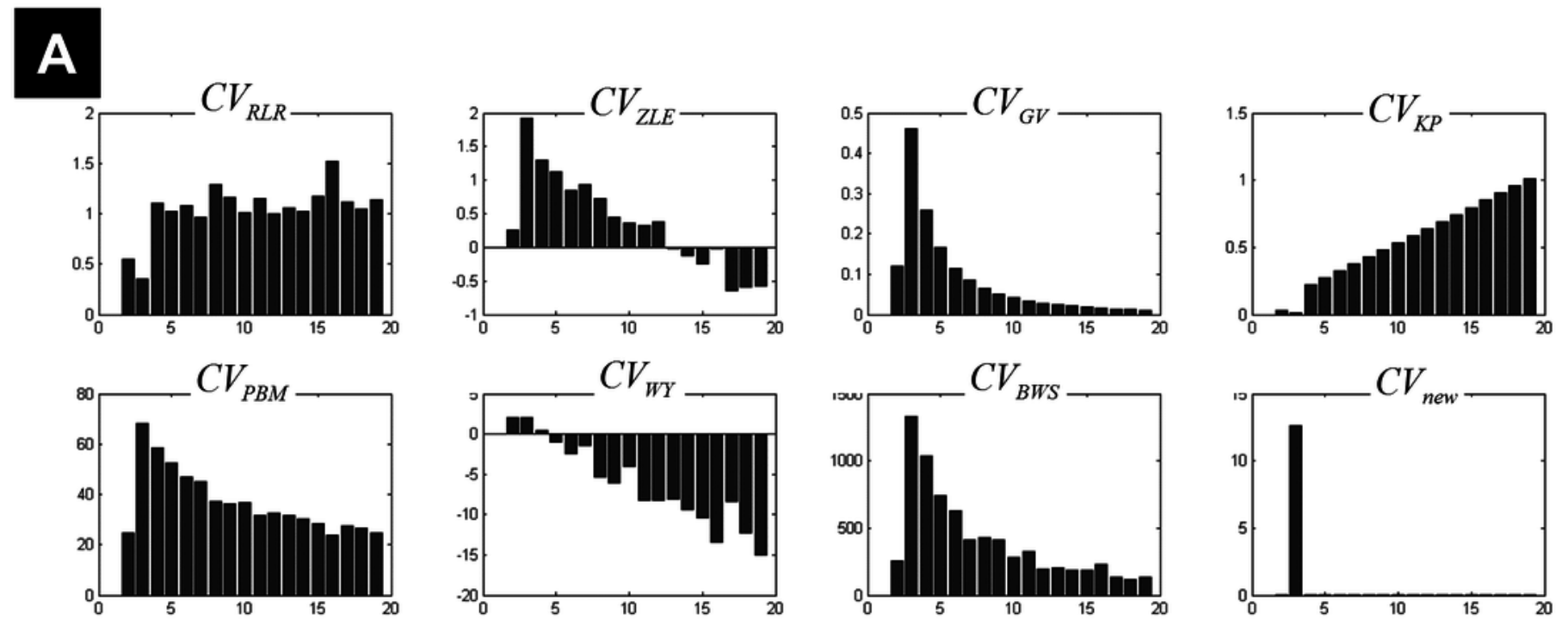

\section{B}
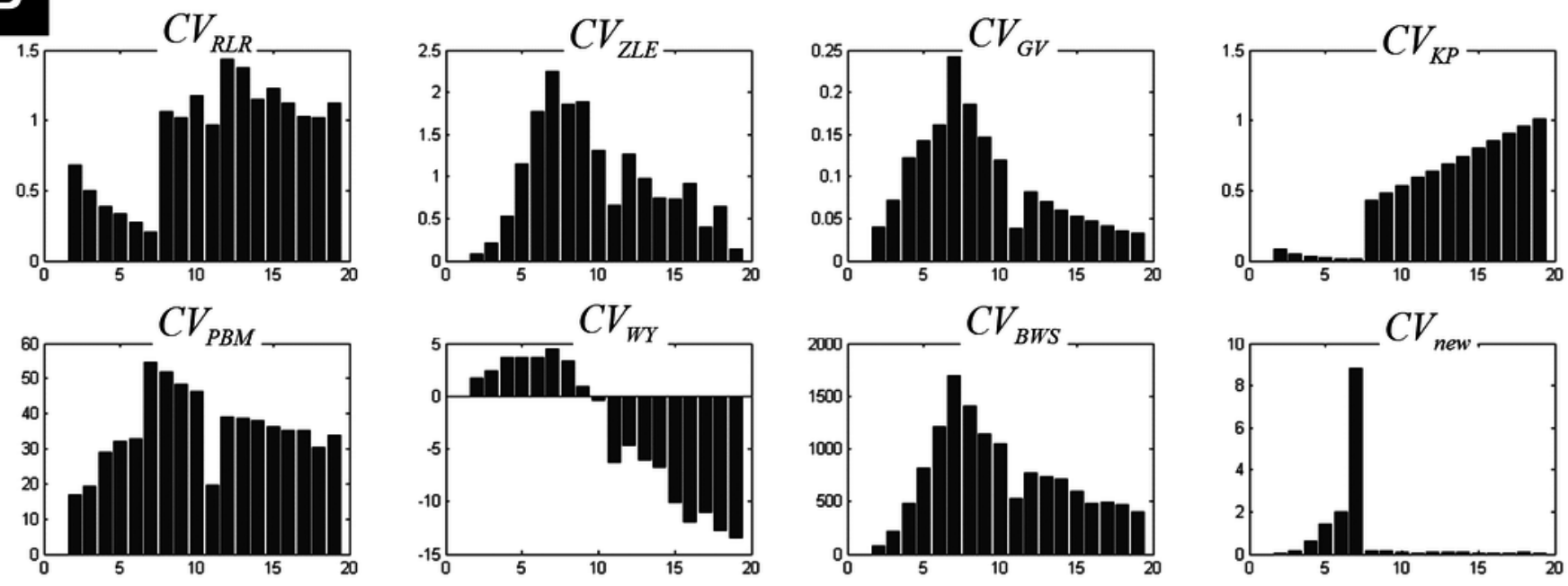

\section{C}
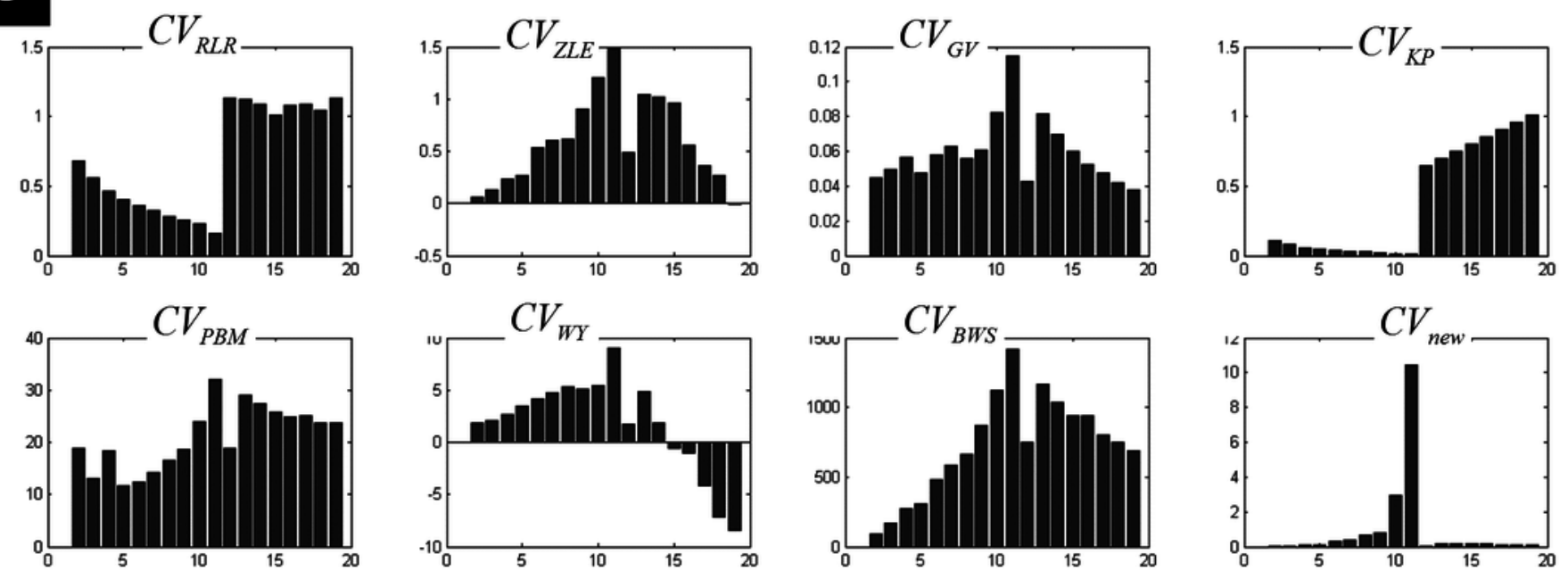


\section{Figure 4}

Plots of the CV indices for the simulated data at high noise level.

Plots of the CV indices for the simulated 3-clusters (A), 7 clusters (B) and 11-clusters (C) with high noise levels $(\sigma=4)$, when the number of clusters increased from 2 to 19. Only the new CV index identified the correct 11-partition at this level of noise. 
A
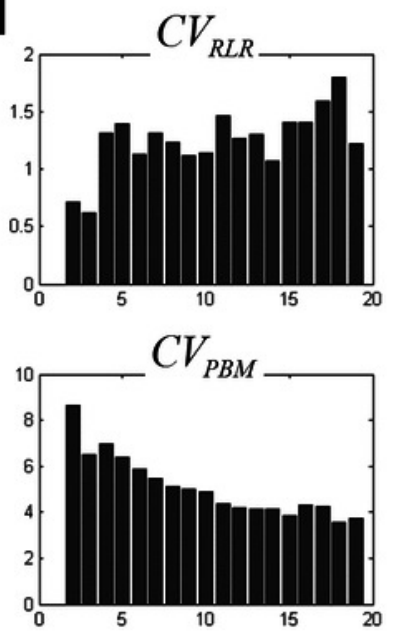

B
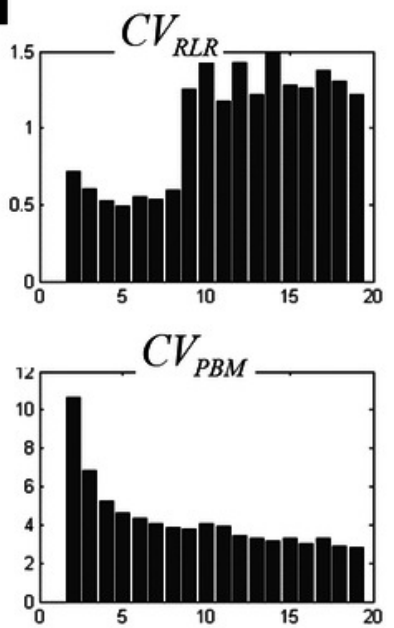

C
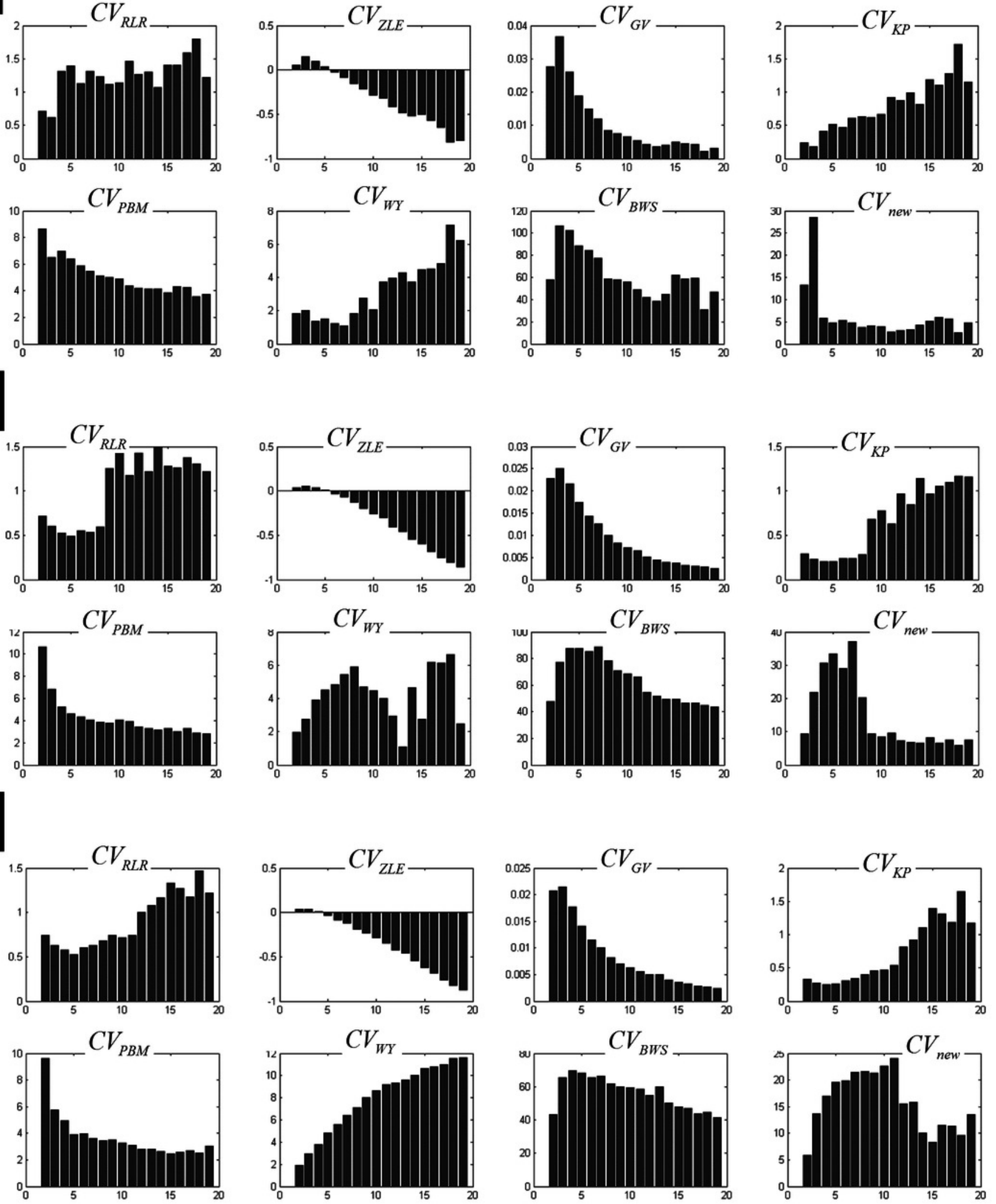


\section{Figure 5}

Illustration of the results using real fMRI data.

(A) Different measures of compactness and separation and (B) the different CV indices. The number of clusters varied from 2 and 39.
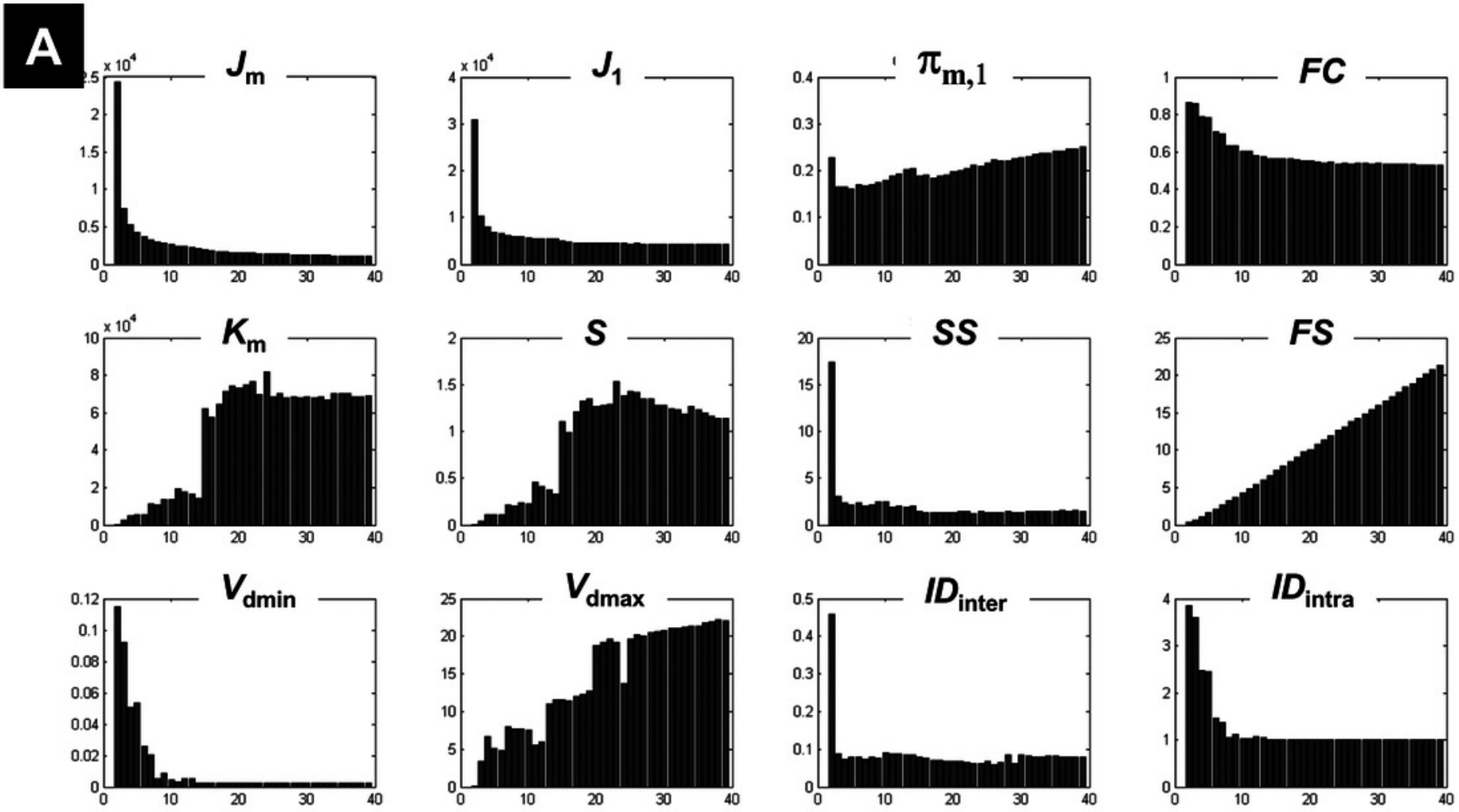

\section{B}
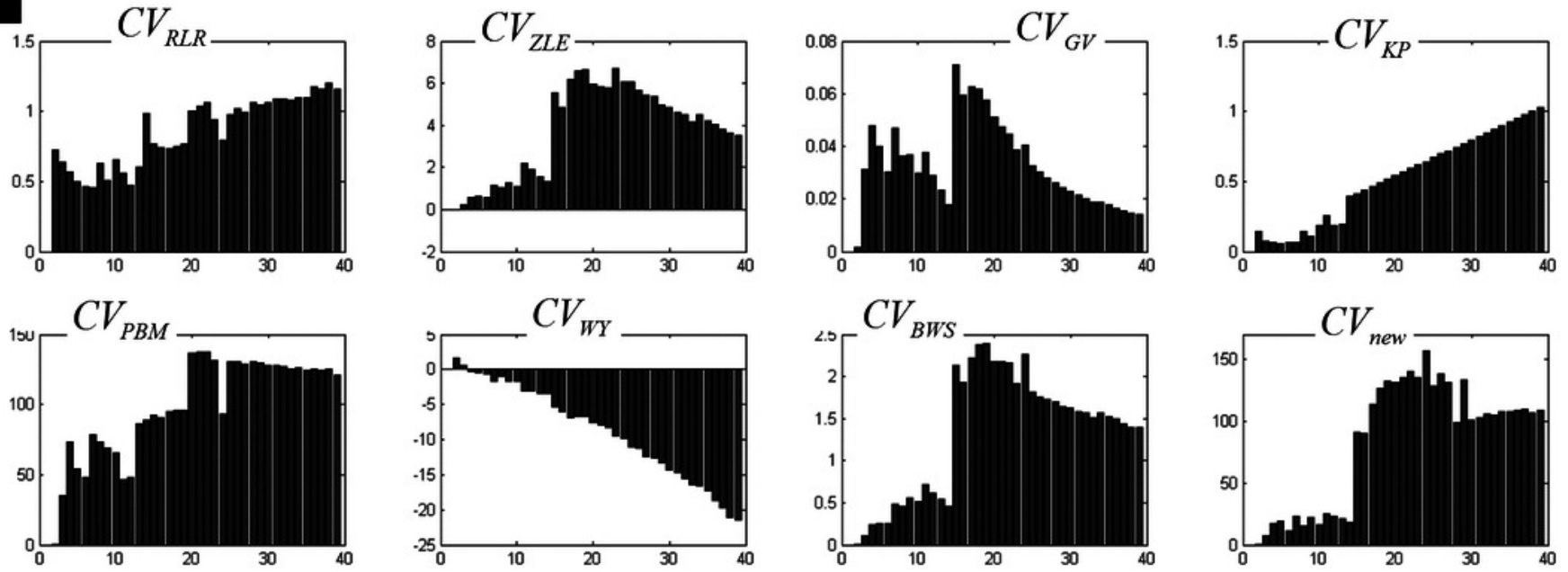


\section{Figure 6}

FCM results at different c values.

(A) Regions' sizes (in number of voxels) for each crisp c-partition (at an arbitrary threshold of $U>0.5$ ). Each dot (diamond shape) represents the size of one region in any cluster of the cpartition (c varying between 2 and 39). A base 10 logarithmic scale is used for the y-axis. (B) Total number of single-voxel regions for each c-partition. For the winning FCM partition ( $c=24$ with $\mathrm{CV}_{\text {new }}$ ), there was less than 4 single-voxel regions per cluster on average. Total number of voxels $n=227,716$; voxel size $=8 \mathrm{~mm}^{3}$. 

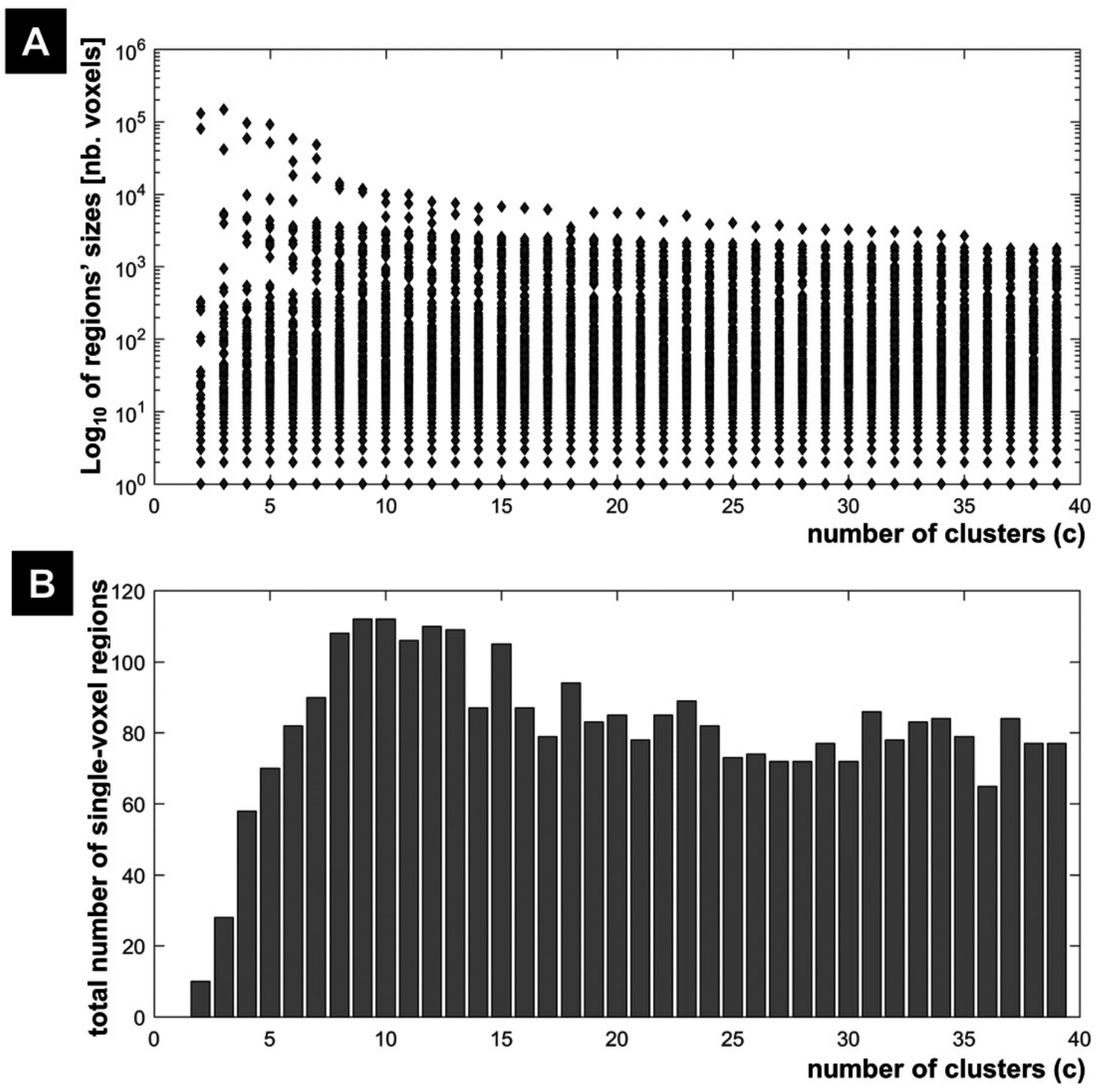


\section{Figure 7}

FCM results at different c values.

FCM results at different $c$ values $(A: C=8, B: C=13, C: C=18$ and $D: C=24)$. Each obtained cluster (a 3D image) of each c-partition is illustrated by its most representative axial slice, with $U$ values varying from 0.1 to 1.0. Cluster label is shown at the top-left corner of each axial slice (in white) and the MNI-z coordinate is indicated in black. For illustration purposes, the cluster that contained the expected activated voxels within the auditory cortex is labelled as Cluster ' 1 '. The scatter plot (E) illustrates the correlations between the centroids of the 24partition and the experimental block design (y-axis) against the fuzzy cardinality (cf.

Equation 6) of each cluster ( $x$-axis). Only one cluster showed significant correlation $(p<0.001)$ with the experimental design $(r=0.7)$. The fuzzy cardinality was divided by the total number of voxels, which would approximately reflect the 'proportion' of voxels contained in each cluster (average proportion around $4 \%(=1 / c)$ ). Using the spatial location of the clustered voxels, one can potentially interpret the results of the FCM 24-partition (D). For example, Cluster 1 is showing auditory activations (cluster of interest) that highly correlated with the experimental block design ( $r=0.7)$; Clusters 2-4 illustrate voxels in the visual system;

Clusters 5-8 illustrate cerebellar and subcortical regions; Clusters 9-10 illustrate different medial parts of the default mode network; Clusters 11-12 contain voxels in ventral brain regions that are prone to MR signal loss; Clusters 13 and 14 are dominated by motion artefacts; Cluster 15 mainly shows CSF voxels; Clusters 19-24 contain white matter voxels. L $=$ left hemisphere, $\mathrm{R}=$ right hemisphere. 

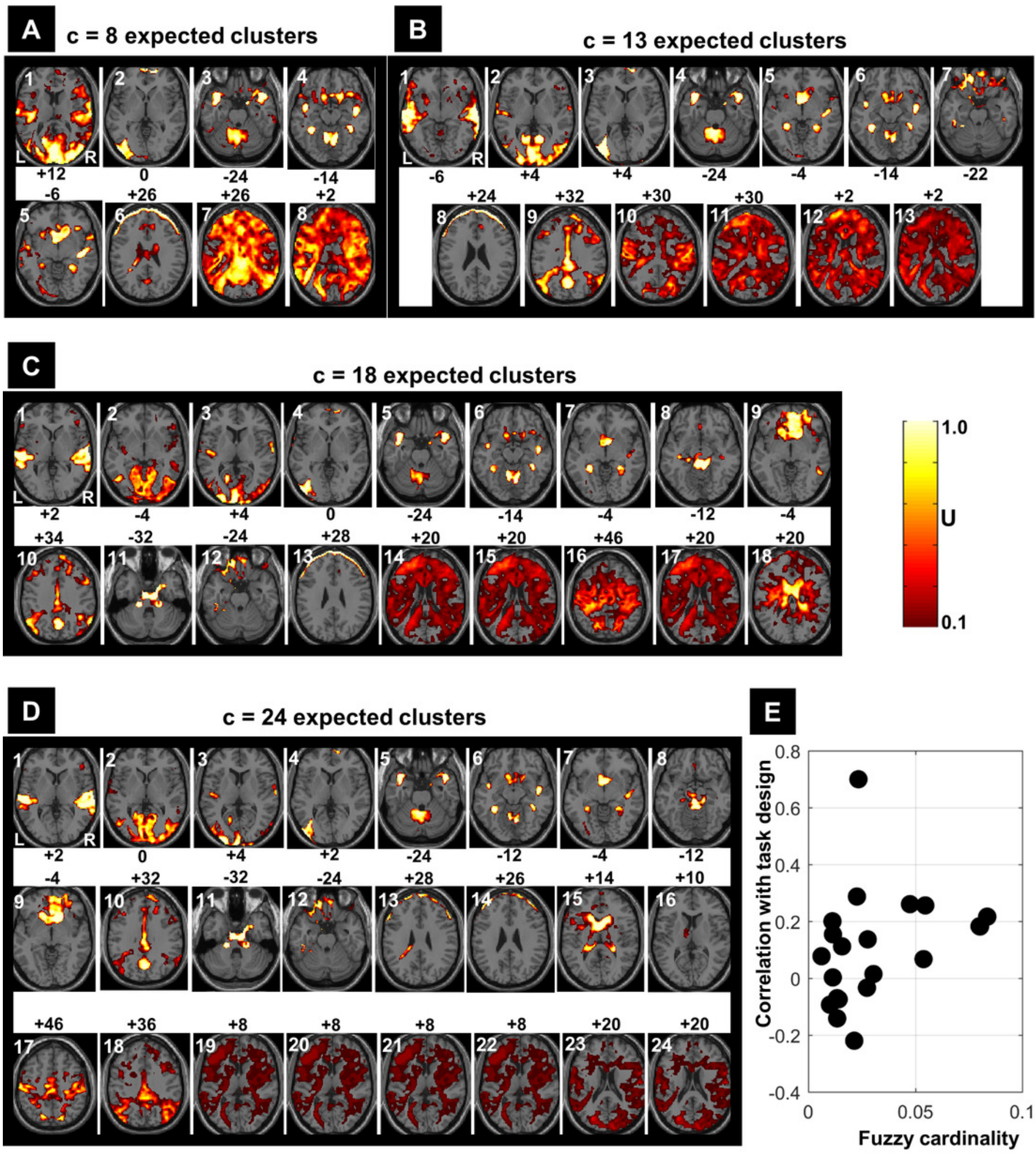


\section{Figure 8}

SPM's results.

SPM results illustrated with the function 'montage' of SPM12, with axial slices varying between $\mathrm{MNI}-\mathrm{Z}=-16 \mathrm{~mm}$ to $\mathrm{MNI}-\mathrm{z}=+36 \mathrm{~mm}$. (A) results at a very liberal threshold of $p<0.05$ uncorrected, $(B)$ at $p<0.05$ FWE-corrected. $L=$ left hemisphere, $R=$ right hemisphere.

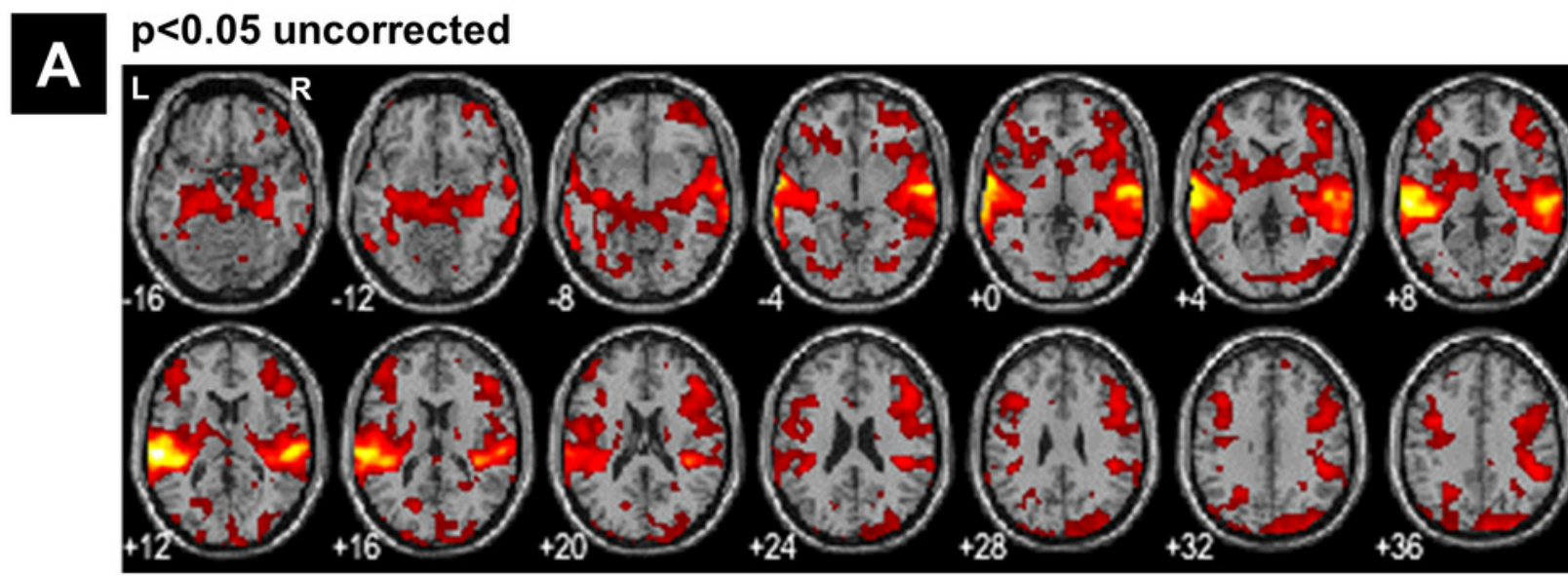

SPM results
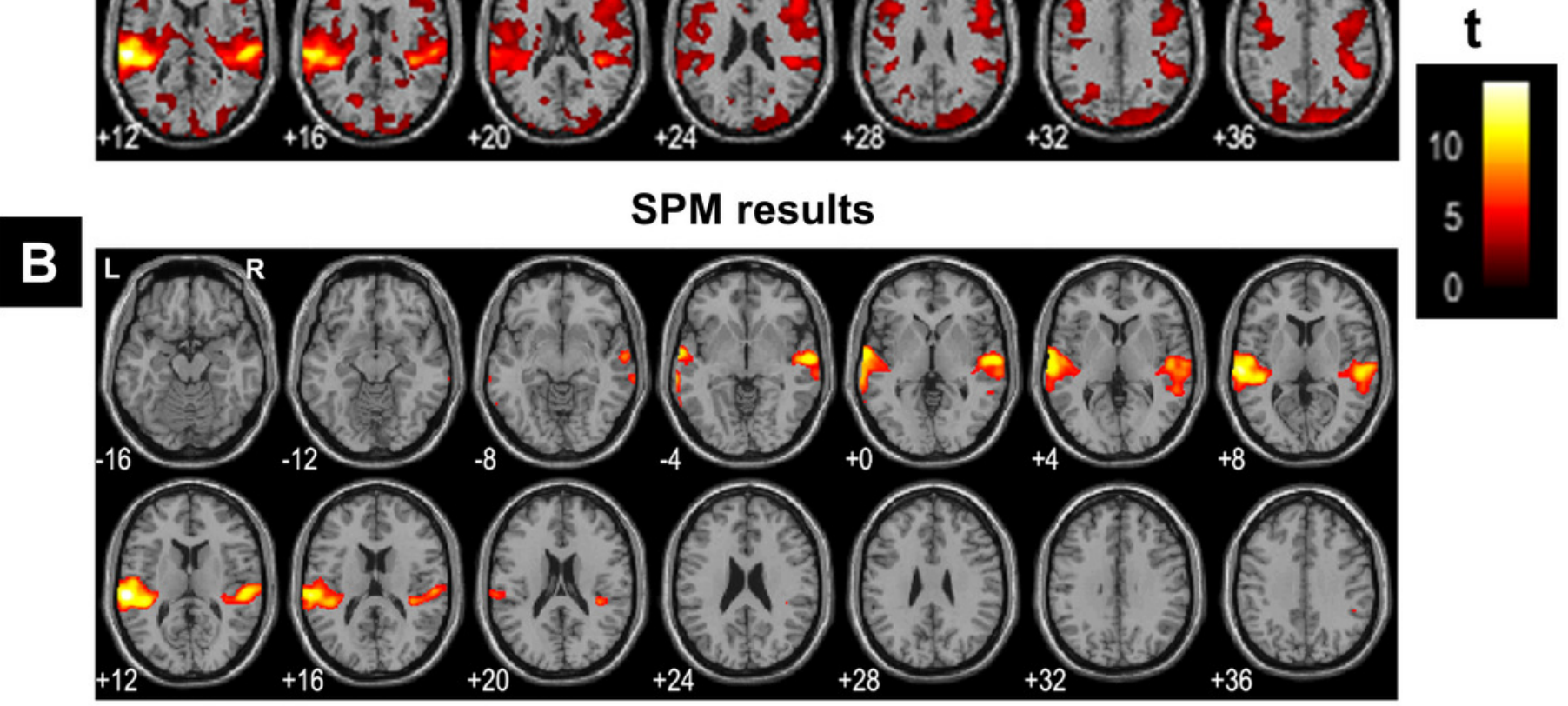

p $<0.05$ FWE-corrected 